\title{
Probing Vibrational Symmetry Effects and Nuclear Spin Economy Principles in Molecular Spin Qubits
}

\author{
Fabio Santanni, ${ }^{\perp}$ Andrea Albino, ${ }^{\perp}$ Matteo Atzori, Davide Ranieri, Enrico Salvadori,* Mario Chiesa, \\ Alessandro Lunghi, Andrea Bencini, Lorenzo Sorace, Federico Totti, and Roberta Sessoli*
}

Cite This: Inorg. Chem. 2021, 60, 140-151

Read Online

\section{ACCESS \\ 山ll Metrics \& More \\ Article Recommendations \\ Supporting Information}

ABSTRACT: The selection of molecular spin qubits with a long coherence time, $T_{\mathrm{m}}$, is a central task for implementing moleculebased quantum technologies. Even if a sufficiently long $T_{\mathrm{m}}$ can be achieved through an efficient synthetic strategy and ad hoc experimental measurement procedures, many factors contributing to the loss of coherence still need to be thoroughly investigated and understood. Vibrational properties and nuclear spins of hydrogens are two of them. The former plays a paramount role, but a detailed theoretical investigation aimed at studying their effects on the spin dynamics of molecular complexes such as the benchmark phthalocyanine $\left(P_{c}\right)$ is still missing, whereas the effect of the latter deserves to be examined in detail for such a class of

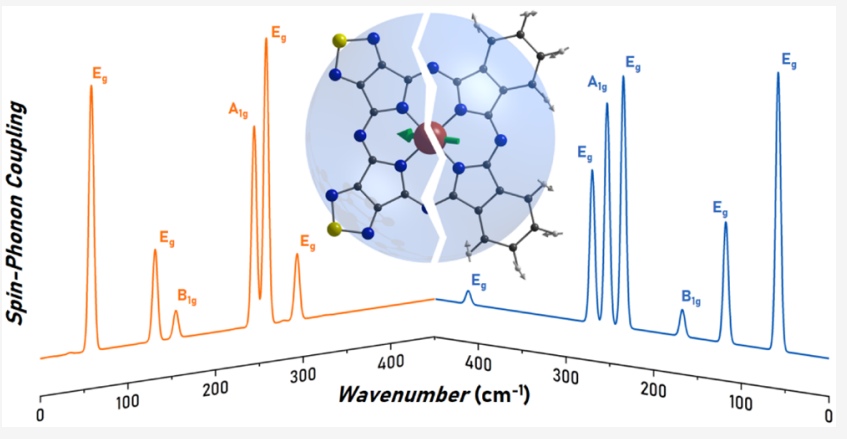
compounds. In this work, we adopted a combined theoretical and experimental approach to investigate the relaxation properties of classical $[\mathrm{Cu}(\mathrm{Pc})]$ and a $\mathrm{Cu}^{\mathrm{II}}$ complex based on the ligand tetrakis(thiadiazole)porphyrazine $\left(\mathrm{H}_{2} \mathrm{TTDPz}\right)$, characterized by a hydrogen-free molecular structure. Systematic calculations of molecular vibrations exemplify the effect of normal modes on the spin-lattice relaxation process, unveiling a different contribution to $T_{1}$ depending on the symmetry of normal modes. Moreover, we observed that an appreciable $T_{\mathrm{m}}$ enhancement could be achieved by removing hydrogens from the ligand.

\section{INTRODUCTION}

Quantum bits, or qubits, represent the fundamental units for the realization of quantum computers. In comparison to their classical analogues, the ultimate advantage of quantum computers arises from using quantum gates as the basic units for logical operations. ${ }^{1}$ This allows solving problems that classical computers cannot solve, reaching the so-called quantum supremacy. ${ }^{2}$ In recent years, different strategies have been employed to integrate qubits in specifically engineered quantum architectures. ${ }^{3}$ However, the realization of universal quantum computers ${ }^{4}$ and fault-tolerant quantum processors $^{5-7}$ can still be considered a fundamental challenge in the field of quantum information science and technology.

Currently, molecular spin qubits, such as $\mathrm{Cu}^{\mathrm{II}}$ and $\mathrm{V}^{\mathrm{IV}}$ complexes, seem to be a realistic alternative to the proposed physical and chemical systems, ${ }^{8-14}$ such as transmons, ${ }^{15,16}$ optical cavities, ${ }^{17}$ and defects in semiconductors and insulators. ${ }^{18-23}$ The reason for this is that, in many cases, they guarantee a long coherence time $\left(T_{\mathrm{m}}\right)$ over a wide range of temperatures, ${ }^{9,12}$ so that the superposition state of two welldefined basis states is accessible and employable for logical operations within algorithms. ${ }^{24}$ In addition, a great advantage is represented by the availability of a set of addressable and accessible electronic and nuclear energy levels that can be probed by electromagnetic pulses. ${ }^{25,26}$
$\mathrm{V}^{\mathrm{IV}} \mathrm{O}$ and $\mathrm{Cu}^{\mathrm{II}}$ complexes with macrocyclic conjugated ligands such as phthalocyanine $\left(\mathrm{H}_{2} \mathrm{Pc}\right){ }^{8,9}$ tetraphenylporphyrin $\left(\mathrm{H}_{2} \mathrm{TPP}\right),{ }^{11,27}$ and their derivatives (e.g., $\mathrm{H}_{2}$ TCPP = $5,10,15,20$-tetrakis (4-carboxyphenyl)porphyrin) ${ }^{11,28}$ have attracted further specific interest in this field because of their excellent features in terms of semiconducting properties, ${ }^{29-31}$ thermal stability, ${ }^{32}$ and surface adsorption. ${ }^{33-36}$ These properties should be compared with those of ad hoc designed molecular systems that either are not thermally stable or are ineligible for chemical processing while showing longer coherence times.

Two main contributions affect the coherence time of phthalocyanine complexes. The first contribution arises from the localized hyperfine interaction between the central ion and the four coordinated nitrogen atoms at the macrocyclic core, while the second contribution stems from the through-space hyperfine interaction with peripheral hydrogen nuclei. ${ }^{8,37}$

Received: August 28, 2020

Published: December 11, 2020 
The first contribution is to be considered intrinsic to this class of molecules and sets the limit of their relaxation properties. ${ }^{37}$ On the other hand, the nuclear spins of hydrogens in phthalocyanine scaffolds might affect electronic coherence through a spectral spin diffusion mechanism. ${ }^{38,39}$ Here, the decoherence efficiency is not a monotonic function of the electron-nuclear distance. When it reaches the critical limit of the spin-diffusion barrier radius, the magnetic interaction is reduced, and the flip-flop transitions of nuclear spins are permitted, thus affecting the coherence time. Following what has been reported in studies conducted on a $\mathrm{V}^{\mathrm{IV}} \mathrm{O}$ series of metal complexes and organic radicals, the critical radius may be reasonably expected to be in the $4-8 \AA$ length scale for molecular spin qubits. ${ }^{38,40}$ Benzylic hydrogens of the Pc ligand, which account for the shortest $\mathrm{Cu}-\mathrm{H}$ distance $(5.89 \AA)$, should then be considered noninnocent (Figure 1).
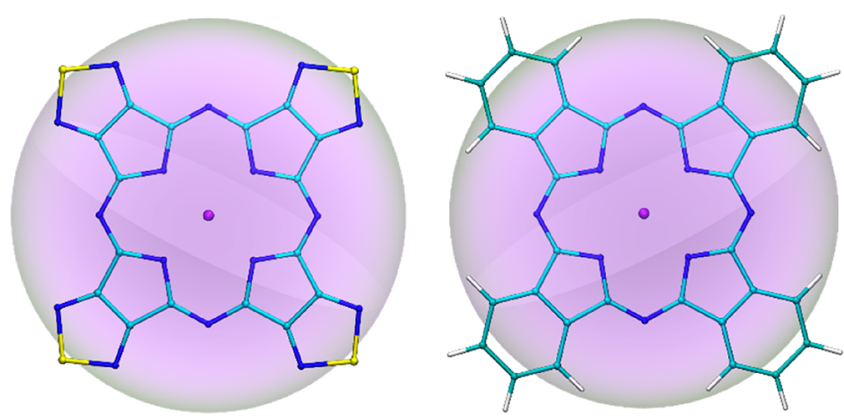

Figure 1. Molecular structures of $[\mathrm{Cu}(\mathrm{TTDP} z)](\text { left })^{51}$ and $[\mathrm{Cu}(\mathrm{Pc})]$ (right). ${ }^{52}$ The violet spheres behind structures highlight the spin diffusion barrier of $6 \AA$. Color code: cyan, C; blue, N; yellow, $S$; purple, $\mathrm{Cu}$.

Some studies aimed at investigating the coherence times of $\alpha-[\mathrm{Cu}(\mathrm{Pc})]$ cast many doubts about spin diffusion barrier effects in this class of macrocyclic complexes. ${ }^{8,37}$ Indeed, when the $\mathrm{Cu}$ iI concentration is lower than $5 \%$ in a diamagnetic isostructural solution, and nuclear-induced decoherence becomes predominant, the main contribution to the spinnuclear interaction was attributed to the hyperfine contact term of the four bonded nitrogen atoms of the ligand rather than to those of the peripheral hydrogen atoms. ${ }^{8}$

Furthermore, the dramatic loss of coherence observed above $100 \mathrm{~K}$ in $[\mathrm{Cu}(\mathrm{Pc})]^{8}$ is mainly due to the rapid decrease in the spin-lattice relaxation time $T_{1}$, limiting the coherence of the system. $T_{1}$ is affected by several contributions arising from a blend of molecular and crystal properties, ${ }^{25}$ i.e. spin-phonon coupling (SPC) and the phonon spectrum, ${ }^{41,42}$ but a detailed vibrational characterization of the isolated molecule can give more subtle insight into the spin-lattice relaxation.

Recent studies have focused on the hydrogen-free macrocyclic ligand tetrakis (thiadiazole)porphyrazine $\left(\mathrm{H}_{2}\right.$ TTDPz $),{ }^{45,46}$ belonging to the Pc family, and the metal complex $[\mathrm{VO}(\mathrm{TTDPz})]^{47}$ revealed that the mean distance between molecules on metal surfaces is lower than that of the phthalocyanine analogue because of the absence of $\mathrm{C}-\mathrm{H}$ bonds on peripheral positions. ${ }^{48,49}$ Furthermore, STM measurements probed that such a feature leads to enhanced exchange interactions between adsorbed molecules. ${ }^{50}$ These previous works inspired us to compare TTDPz and Pc systems to understand the effect of spin-active nuclei on the coherence time of molecular qubits, which is still under active investigation. ${ }^{43,44}$ Therefore, we present here a combined experimental and theoretical study on the vibrational and coherence properties of the $\mathrm{Cu}^{\mathrm{II}}$ complex $[\mathrm{Cu}(\mathrm{TTDPz})]$ compared to those of the structurally similar but hydrogen-rich $[\mathrm{Cu}(\mathrm{Pc})]$. We expect that, given the lower magnetic moment of nitrogen nuclei compared to hydrogen ones and the almost nuclear spin free nature of sulfur atoms, the decoherence induced by the ligand should be reduced in this complex in comparison to $[\mathrm{Cu}(\mathrm{Pc})]$.

Our results - based on pulsed EPR, ac susceptibility, and $a b$ initio calculations of molecular vibrations and their SP coupling-allowed us to pinpoint the differences and peculiarities of these two systems.

\section{RESULTS}

Synthesis and Structural Characterization. The methods used in this work to obtain $\mathrm{H}_{2} \mathrm{TTDPz}$ and its complexes are based on previously reported synthetic strategies, appropriately adapted to the current case (see Synthesis Procedures in the Supporting Information). The ligand $\mathrm{H}_{2} \mathrm{TTDPz}$ was obtained according to a method developed by Linstead et al. ${ }^{53}$ It relies on obtaining at first the magnesium complex $[\mathrm{Mg}(\mathrm{TTDPz})]$, which is then treated with a strong acid $\left(\mathrm{CF}_{3} \mathrm{COOH}\right)$ to achieve the free ligand $\mathrm{H}_{2} \mathrm{TTDPz}\left(\mathrm{H}_{2} \mathbf{L}\right) .{ }^{46}$ Even though this procedure can be used to obtain both the $\mathrm{Cu}^{\mathrm{II}}$ complex $[\mathrm{Cu}(\mathrm{TTDPz})](\mathbf{1})$ and its $\mathrm{Ni}^{\mathrm{II}}$ diamagnetic analogue $[\mathrm{Ni}(\mathrm{TTDPz})]$, basically by reacting $\mathrm{H}_{2} \mathrm{~L}$ with the acetate or chloride salt of the target metal, ${ }^{46,54}$ we found it more convenient to obtain compound $\mathbf{1}$ directly by templating its formation around the $\mathrm{Cu}^{\mathrm{II}}$ ion, as previously reported. $^{45}$

All reported compounds based on this ligand are almost insoluble in all common organic solvents, but they show an appreciable solubility in strong acids, such as $\mathrm{H}_{2} \mathrm{SO}_{4}$ and methanesulfonic acid $\left(\mathrm{CH}_{3} \mathrm{SO}_{3} \mathrm{H}\right)$. The treatment of these compounds with these strong acids could lead to small contaminations of paramagnetic impurities. In the case of $[\mathrm{Ni}(\mathrm{TTDPz})]$, for example, the paramagnetic fraction after treatment with $\mathrm{CH}_{3} \mathrm{SO}_{3} \mathrm{H}$ was estimated to be $0.25 \%$ by direct current magnetometry, and it has been assigned to the presence of paramagnetic $\mathrm{Ni}^{\mathrm{II}}$ in an octahedral coordination environment (Figure S1). Magnetic dilution was then achieved by using the free ligand $\mathrm{H}_{2} \mathrm{~L}$, as for the case of $[\mathrm{Cu}(\mathrm{Pc})](2),{ }^{8}$ to reduce the paramagnetic contribution to the $\mathrm{Cu}^{\mathrm{II}}$ decoherence. Moreover, $\mathrm{H}_{2} \mathrm{~L}$ crystals do not show the typical polymorphism encountered in other compounds of the same class. Indeed, they can be obtained only as an $\alpha$-phase polymorph, which is the same molecular packing encountered in $\alpha$-phase $[\mathrm{Cu}(\mathrm{Pc})] .{ }^{51} \mathrm{CH}_{3} \mathrm{SO}_{3} \mathrm{H}$ was thus adopted as the solvent to obtain magnetic dilutions of $\mathbf{1}$ in $\mathrm{H}_{2} \mathrm{~L}, 2 \%\left(\mathbf{1}_{2 \%}\right)$ and $0.1 \%\left(\mathbf{1}_{0.1 \%}\right)$, and of $\mathbf{1}$ in $[\mathrm{Ni}(\mathrm{TTDPz})], 20 \%\left(\mathbf{1}_{20 \%}\right)$ (see Experimental and Theoretical Details and the Supporting Information). The same strategy involving $\mathrm{CH}_{3} \mathrm{SO}_{3} \mathrm{H}$ as the solvent was also employed to get the magnetic diluted (in $\left.\mathrm{H}_{2} \mathrm{Pc}\right) \alpha$-phases $\left[\mathrm{Cu}_{0.2}(\mathrm{Pc})\right]\left(\mathbf{2}_{\mathbf{2 0} \%}\right)$ and $\left[\mathrm{Cu}_{0.001}(\mathrm{Pc})\right]\left(\mathbf{2}_{\mathbf{0 . 1}}\right)$. Direct current (DC) magnetic characterizations of $\mathbf{1}_{20 \%}$ and $\mathbf{2}_{\mathbf{2 0} \%}$ are reported in Figures S2 and S3.

Because of the limited stability of compounds in acid solutions, $\mathrm{H}_{2} \mathrm{SO}_{4}$ or $\mathrm{CH}_{3} \mathrm{SO}_{3} \mathrm{H}$ cannot be used to obtain large crystals suitable for single-crystal $\mathrm{X}$-ray diffraction. Therefore, all compounds were obtained as microcrystalline powders and structurally characterized by powder X-ray diffraction analysis (PXRD). Thus, the collected patterns were compared with 
those simulated from atomic coordinates reported in the literature (Figures S4-S6). ${ }^{51,52}$ The PXRD measurements revealed that compound $\mathbf{1}$ was obtained as a microcrystalline solid which crystallizes in the $\gamma$-phase (Figure S4), while compound $[\mathrm{Ni}(\mathrm{TTDPz})]$ crystallized in a different mesophase with reference to those reported in the literature. ${ }^{51}$ A PXRD analysis confirms that compounds $\mathbf{2}_{20 \%}$ and $\mathbf{2}_{0.1 \%}$ were both obtained as pure $\alpha$-phase polymorphs (Figure S6). ${ }^{52}$ All of the other diluted compounds were obtained as amorphous solids and investigated as such.

Continuous-Wave Electron Paramagnetic Resonance. The static magnetic properties of compounds $\mathbf{1}, \mathbf{1}_{\mathbf{2 0}}, \mathbf{1}_{\mathbf{2}}$, $\mathbf{1}_{0.1 \%}, \mathbf{2}_{20 \%}$, and $\mathbf{2}_{0.1 \%}$ were investigated by continuous-wave electron paramagnetic resonance (CW-EPR) spectroscopy at the X-band $(v \approx 9.4 \mathrm{GHz})$ on microcrystalline powder samples. The EPR spectrum of $\mathbf{1}$ was recorded at room temperature, while the other spectra were taken at $T=30 \mathrm{~K}$. The spectra (Figures S7 and S8) show the characteristic hyperfine structure due to coupling of the electronic spin $S=$ $1 / 2$ of the $\mathrm{Cu}^{\mathrm{II}}$ ion with the nuclear spin $I=3 / 2$ of both copper isotopes ${ }^{63} \mathrm{Cu}$ (natural abundance $(\mathrm{NA})=69.17 \%$ ) and ${ }^{65} \mathrm{Cu}(\mathrm{NA}=30.83 \%) .{ }^{55,56}$ It is evident that the superhyperfine interaction with the four ${ }^{14} \mathrm{~N}$ atoms $(I=1)$ of the macrocyclic ligand is more resolved by increasing the dilution. A complete characterization of the magnetic anisotropy of the samples was accomplished by simulating the spectra ${ }^{57}$ with the following spin Hamiltonian

$$
\hat{H}_{\mathrm{s}}=\mu_{\mathrm{B}} \mathbf{B} \cdot \mathbf{g} \cdot \hat{S}+\hat{S} \cdot \mathbf{A}^{\mathrm{Cu}} \cdot \hat{I}^{\mathrm{Cu}}+\sum_{i=1}^{4} \hat{S} \cdot \mathbf{A}_{i}^{\mathrm{N}} \cdot \hat{I}^{\mathrm{N}}
$$

where $\mathbf{g}$ is the tensor characterizing the electronic Zeeman interaction, while $\mathrm{A}^{\mathrm{Cu}}$ and $\mathrm{A}^{\mathrm{N}}$ describe the interaction of the $\mathrm{Cu}^{\mathrm{II}}$ electronic spin with its nuclear spin and those of the four bonded nitrogen atoms, respectively. For compound $\mathbf{1}$, the $\mathrm{A}^{\mathrm{Cu}}$ tensor was assumed to be axial, which is analogous to previous literature reports on $2 .{ }^{56}$ Furthermore, the four $\mathbf{A}^{\mathrm{N}}$ tensors were assumed to be identical and axial, with the local $z$ axis oriented along the $\mathrm{N}-\mathrm{Cu}$ bond. All simulations were implemented by adopting a Voigtian line shape, obtained as a convolution of a Gaussian and a Lorentzian distribution with the same line width. The best simulation parameters are given in Table S1. The spectra obtained for $\mathbf{2}_{20 \%}$, and $\mathbf{2}_{0.1 \%}$ are interpreted and simulated along the same lines. In this case, simulations were implemented by adopting a set of parameters reported in the literature (Figure S8). ${ }^{56}$ Compounds $\mathbf{1}_{\mathbf{2} \%}$ and $\mathbf{1}_{0.1 \%}$ were also characterized via CW-EPR and echo detected field swept (EDFS) spectroscopy at the Q-band $(v \approx 34 \mathrm{GHz})$ (Figure 2). The simulations of the spectra recorded at the $\mathrm{Q}$ band were implemented using the same set of spin Hamiltonian parameters (Table 1) as for X-Band spectra and by only adjusting the line width and the strain contributions, which are frequency-dependent. Moreover, all EPR spectra exhibit a signal due to a radical impurity fraction, separated from the other features at Q-band frequencies. According to the Q-band spectral simulations, the radical percentage varies from $0.005 \%$ to $0.01 \%$ of the $\mathrm{Cu}^{\mathrm{II}}$ amount, analogously to what has been found for porphyrins. ${ }^{58}$

As a further point, the presence of an intense spin-echo indicates that quantum coherence should be observed for 1 up to at least $30 \mathrm{~K}$. Indeed, the EDFS spectrum obtained by integrating the Hahn echo as a function of the applied magnetic field corresponds to the absorption of the CW

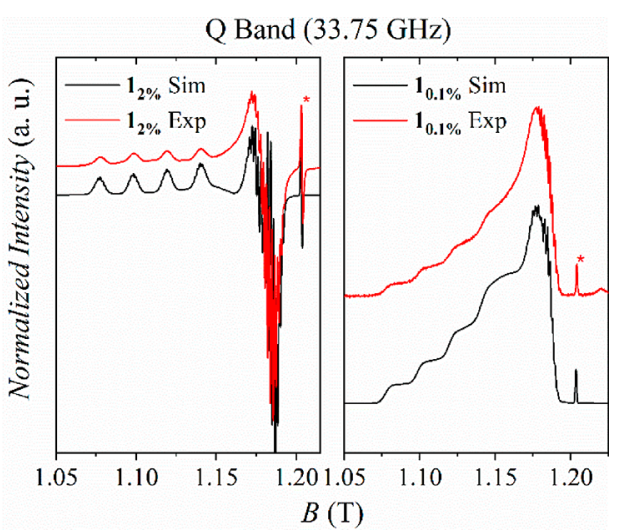

Figure 2. Experimental CW-EPR spectrum of $\mathbf{1}_{2 \%}$ (left) and EDFS spectrum of $\mathbf{1}_{0.1 \%}$ (right) reported with the respective simulated spectra. The radical impurity signal $(g=2.0)$ is highlighted with an asterisk.

spectrum and demonstrates that coherence is created throughout the entire spectrum.

Calculation of the spin Hamiltonian parameters was carried out at the DFT level. The molecular geometry used for these simulations was obtained by a periodic DFT optimization (see Experimental and Theoretical Details). The results show good agreement with experimental CW EPR spectra with collinear $\mathbf{g}$ and $\mathbf{A}$ tensors.

The two compounds show similar magnetic properties since they are characterized by a singly occupied molecular orbital (SOMO) mainly constituted by the $\mathrm{d}_{x^{2}-y^{2}}$ orbital (Figure 3 ). The $\mathrm{d}$ orbitals representations are, following the energy ladder from the lowest to the highest in energy, $\mathrm{b}_{2 \mathrm{~g}}\left(\mathrm{~d}_{x y}\right) \mathrm{e}_{\mathrm{g}}\left(\mathrm{d}_{x z}, \mathrm{~d}_{y z}\right)$, $a_{1 g}\left(d_{z}^{2}\right)$, and $b_{1 g}\left(d_{x}^{2}-y^{2}\right)$.

Alternating Current Susceptibility Measurements. A common way to investigate the magnetization dynamics is by alternating current (AC) susceptometry. ${ }^{59}$ Following the model of Casimir and Du Prè, ${ }^{60}$ the magnetization of a spin system in the presence of an oscillating magnetic field depends on the capability of the spin ensemble to exchange energy with the thermal bath, and it is thus directly correlated to the spinlattice interaction. This relaxation process is characterized by a time constant $\tau$ (relaxation time), which is temperature (T) and magnetic field $(B)$ dependent.

The AC susceptibilities of $\mathbf{1}_{\mathbf{2 0} \%}$ and $\mathbf{2}_{\mathbf{2 0}}$ were probed in the frequency range $10 \mathrm{~Hz}$ to $10 \mathrm{kHz}$ by sweeping $T$ in the range $2.5-30 \mathrm{~K}$ at different $B$ values (Figures S9-S13) and in the same frequency range by sweeping $B$ from 0 to $8.5 \mathrm{~T}$ at different temperatures (Figures S14-S17). The $\tau$ values were extracted by fitting the frequency dependence of the imaginary component, $\chi^{\prime \prime}$, using a generalized Debye model, ${ }^{61}$ which considers a distribution of relaxation times. In Figure 4, we report a comparison between the $\tau$ values observed for $\mathbf{1}_{\mathbf{2 0} \%}$ and for $\mathbf{2}_{\mathbf{2 0} \%}$ as a function of $B$ at $T=5 \mathrm{~K}$ and as a function of $T$ when $B=1 \mathrm{~T}$ (the complete set of data for the two derivatives is reported in Figures S18 and S19).

The general temperature and field dependences of $\tau$ for the two complexes are similar, even though the magnetization of compound $\mathbf{2}_{\mathbf{2 0} \%}$ relaxes faster than that of $\mathbf{1}_{\mathbf{2 0} \%}$ under all the investigated experimental conditions (Figure 4). As B increases, the relaxation time of $\mathbf{1}_{\mathbf{2 0} \%}$ and $\mathbf{2}_{\mathbf{2 0} \%}$ increases until it reaches a maximum between 1.7 and $1.9 \mathrm{~T}$, in analogy with previous observations on other $S=1 / 2$ systems. ${ }^{9,11}$ This nonmonotonous behavior can be elucidated by considering 
Table 1. Comparison among Parameters Obtained from the Simulations of the Experimental EPR Spectra of Compounds $\mathbf{1}_{2 \%}$ and $1_{0.1 \%}$, Calculated $g$ Values for 1 and 2, and Experimental Parameters Reported in Ref $56^{a}$

\begin{tabular}{lllcrrrrrr} 
& \multicolumn{1}{c}{$g_{x}$} & \multicolumn{1}{c}{$g_{y}$} & \multicolumn{1}{c}{$g_{z}$} & $A_{x}^{\mathrm{Cu}}(\mathrm{MHz})$ & $A_{y}^{\mathrm{Cu}}(\mathrm{MHz})$ & $A_{z}^{\mathrm{Cu}}(\mathrm{MHz})$ & $A_{x^{\prime}}^{\mathrm{N}}(\mathrm{MHz})$ & $A_{y^{\prime}}^{\mathrm{N}}(\mathrm{MHz})$ & $A_{z^{\prime}}^{\mathrm{N}}(\mathrm{MHz})$ \\
$\mathbf{1}_{\mathbf{2}^{\circ}{ }^{b}}$ & $2.043(1)$ & $2.043(1)$ & $2.172(1)$ & $69(2)$ & $69(2)$ & $630(1)$ & $45(2)$ & $45(2)$ & $51(2)$ \\
$\mathbf{1}_{\mathbf{0 . 1}}{ }^{b}$ & $2.043(1)$ & $2.043(1)$ & $2.172(1)$ & $69(2)$ & $69(2)$ & $630(1)$ & $45(2)$ & $45(2)$ & $51(2)$ \\
$\mathbf{1}^{c}$ & 2.044 & 2.044 & 2.146 & 30 & 30 & 663 & 44 & 46 & 57 \\
$\mathbf{2}^{c}$ & 2.042 & 2.042 & 2.134 & 31 & 31 & 671 & 45.2 & 46.5 \\
$\mathbf{2}^{d}$ & $2.0390(5)$ & $2.0390(5)$ & $2.1577(5)$ & $83(3)$ & $83(3)$ & $648(3)$ & $44.7(2)$ & $45.4(2)$ & 57.7
\end{tabular}

${ }^{a}$ The local reference frame for nitrogen atoms $\left(x^{\prime} y^{\prime} z^{\prime}\right)$ was chosen with the $z^{\prime}$ direction along the $\mathrm{Cu}-\mathrm{N}$ bond. ${ }^{b}$ Experimental data. ${ }^{c} \mathrm{Calculated}$ data. ${ }^{d}$ Data extracted from ref 56 and adopted for the simulations reported in Figure S8 for $\mathbf{2}_{\mathbf{2 0} \%}$ and $\mathbf{2}_{\mathbf{0} . \mathbf{1} \%}$.

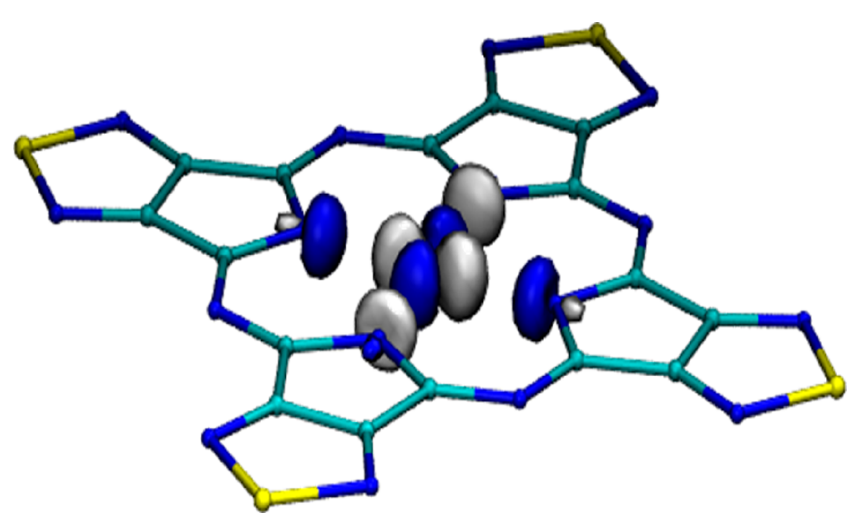

Figure 3. Representative sketch of compound 1 showing the DFT calculated SOMO UNO orbital (isodensity surface value of 0.08 e/ $\mathrm{au}^{3}$ ). Color code: cyan, $\mathrm{C}$; blue, $\mathrm{N}$; yellow, $\mathrm{S}$; purple, $\mathrm{Cu}$ (covered by orbital white and blue lobes).

that the magnetic field suppresses the effect of both hyperfine and electronic spin dipolar interactions, thus increasing the relaxation time. At the same time, the application of the magnetic field increases the effect of the direct relaxation mechanism, which scales as $B^{4}{ }^{62}$

For a more detailed comparison, we fit the $B$-dependent behavior of $\tau$ with the phenomenological model of Brons and Van Vleck to extract the values of the empirical parameters from eq 2 . $^{63,64}$

$$
\tau^{-1}=a B^{4}+d \frac{\left(1+e x^{2}\right)}{\left(1+f x^{2}\right)}
$$

It is evident that, in our case, this model leads to an overestimation of the $\tau$ values below $B=0.1 \mathrm{~T}$ (Figure 4), where the effect of the direct term is negligible.

An analysis of the temperature-dependent behavior of $\mathbf{1}_{\mathbf{2 0}} \%$ and $\mathbf{2}_{20 \%}$ leads to the observation of the same behavior reported for similar macrocyclic complexes of $S=1 / 2$ ions. $^{11}$ Indeed, the $\tau$ values of both compounds at low temperatures depend on temperature as $\tau \propto T^{-n}$ with an exponent $n \approx 0.7$, which differs from $n=1$ expected for a pure direct mechanism. ${ }^{62}$

The AC susceptibility was also probed on pure $\mathbf{1}$ and $\mathbf{2}$ in order to highlight the effect of electron spin coupling on the relaxation time of concentrated samples. The plots of AC susceptibility data obtained at $T=7.5 \mathrm{~K}$ with a variation of $B$ in the range $0-4 \mathrm{~T}$ are reported in Figure S20. Pure samples show a fast relaxation dynamic, which cannot be probed in the experimental frequency range. Moreover, the observed increment in relaxation rate is more relevant for 1 than for 2 . Such an effect may be attributed to a stronger coupling in $\mathbf{1}$ due to the presence of polarizable sulfur atoms that drive the interaction between neighboring units in the crystal lattice.

Pulsed EPR Spectroscopy. Pulsed EPR spectroscopy was adopted to investigate the coherence time of the system $\left(T_{\mathrm{m}}\right)$ and its spin-lattice relaxation time $\left(T_{1}\right)$, as it directly guarantees access to the spin dynamics of the system. ${ }^{65}$

The coherence time, $T_{\mathrm{m}}$, as a function of temperature was measured for $\mathbf{1}_{\mathbf{0 . 1}}$ and $\mathbf{2}_{\mathbf{0 . 1}}$ polycrystalline powder samples in the $4.3-150 \mathrm{~K}$ range at the Q-band frequency and in a magnetic field corresponding to the maximum echo intensity (i.e., probing molecules with their unique axis perpendicular to the magnetic field). The echo decay traces were fitted using
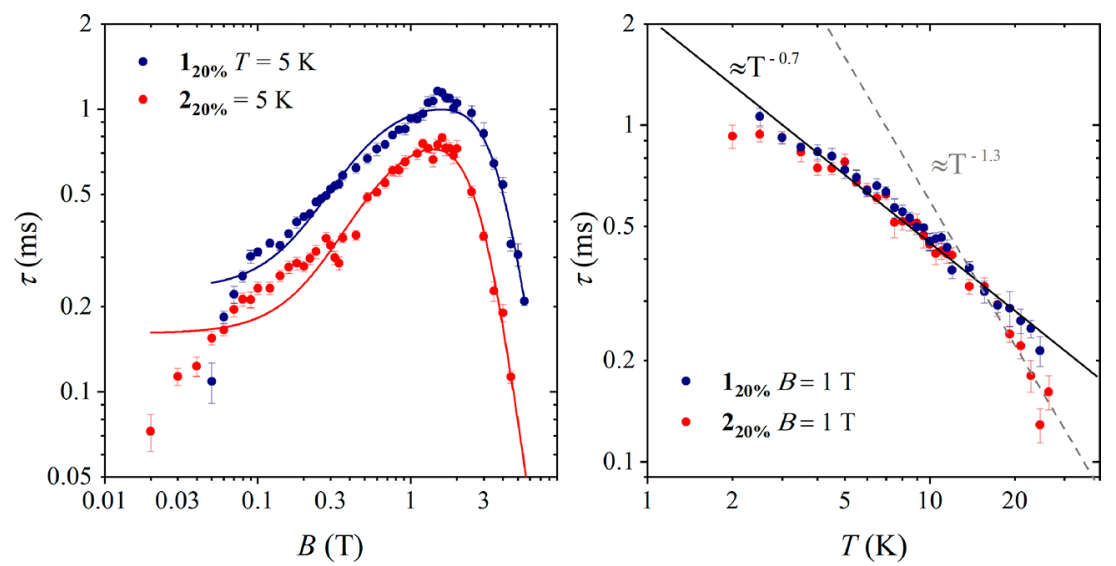

Figure 4. Comparison between the experimental $\tau(\mathrm{ms})$ values obtained for $\mathbf{1}_{\mathbf{2 0} \%}$ and $\mathbf{2}_{\mathbf{2 0} \%}$. The relaxation times are reported in a log-log plot as a function of $B(\mathrm{~T})$ at $T=5 \mathrm{~K}$ on the left side and as a function of $T(\mathrm{~K})$ at $B=1 \mathrm{~T}$ on the right side. Solid lines represent the best fits obtained by using models reported in the main text. The broken line represents the power law that best reproduces the high temperature data of 2 . 
both a stretched-exponential and a biexponential equation, giving qualitatively similar results (Figure S21). The thermal variation of $T_{\mathrm{m}}$ (Figure 5) shows an almost temperature-

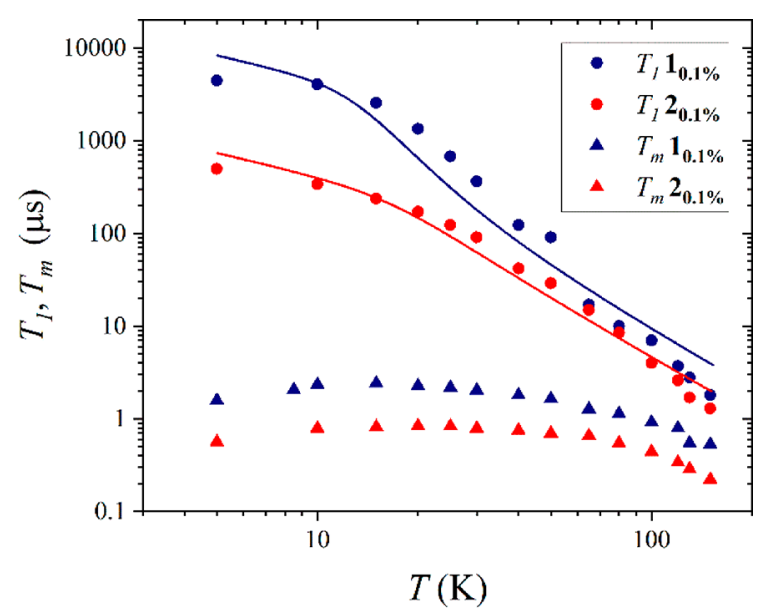

Figure 5. Comparison between $T_{1}$ and $T_{\mathrm{m}}$ values extracted from experimental measurements conducted on $\mathbf{1}_{0.1 \%}$ and $\mathbf{2}_{0.1 \%}$. Both $T_{1}$ and $T_{\mathrm{m}}$ values were obtained by a stretched-exponential fit of the experimental traces. Data obtained using a biexponential fitting are reported in Figure S21 in the Supporting Information. Solid lines represent the best fit obtained by using eq 4 .

independent behavior in the $4.3-50 \mathrm{~K}$ range, with values on the order of $1 \mu \mathrm{s}$, falling to about $0.5 \mu \mathrm{s}$ at $150 \mathrm{~K}$. Furthermore, the $T_{\mathrm{m}}$ value of $\mathbf{1}_{0.1 \%}$ is about $1 \mu \mathrm{s}$ longer than that of $\mathbf{2}_{0.1 \%}$ at all the investigated temperatures. Both $\mathbf{1}_{0.1 \%}$ and $\mathbf{2}_{0.1 \%}$ present a peculiar behavior at low $T$, where $T_{\mathrm{m}}$ has a maximum between 15 and $20 \mathrm{~K}$.
The observed downturn of $T_{\mathrm{m}}$ at $T<20 \mathrm{~K}$ has been already observed in other systems, ${ }^{66}$ in which it has been attributed to a switch of the dynamic regime, i.e. from a $T_{1}$-limited regime to one affected by the rotation of methyl groups. The proposed explanation does not seem to be applicable here because (i) there is a lack of methyl groups or other substituents with rotational degrees of freedom and (ii) the $T_{1}$-limited regime becomes more relevant at temperatures higher than those involved here.

A direct comparison between the $T_{\mathrm{m}}$ values observed for $\mathbf{1}_{\mathbf{0 . 1} \%}$ and $\mathbf{1}_{\mathbf{2} \%}$ (Figure S22) shows that stronger spin-spin interactions lead to a noticeable loss of coherence, confirming previous reports on $[\mathrm{Cu}(\mathrm{Pc})]{ }^{8}$ Moreover, the observation of Rabi oscillations in $\mathbf{1}_{\mathbf{0 . 1}}$ up to $50 \mathrm{~K}$ (Figure 6) guarantees control of coherent spin manipulation on this system.

Inversion recovery experiments were performed to investigate the temperature dependence of $T_{1}$. The experimental traces were fit with a stretched-exponential function, and the corresponding $T_{1}$ values are plotted in Figure 5. The full set of $T_{1}$ and $T_{\mathrm{m}}$ values as a function of $T$ are reported in Table S2.

The fit of $T_{1}$ as a function of temperature is commonly performed using the phenomenological equation (3). ${ }^{39}$

$$
T_{1}^{-1}=a_{\mathrm{dir}} T^{x}+a_{\mathrm{Ram}} T^{n}
$$

where $a_{\mathrm{dir}}$ and $a_{\mathrm{Ram}}$ are two weight constants that define the relative contributions of direct and Raman processes to the relaxation, $x$ is usually equal to 1 , and $n$ can vary in the range 2-9 depending on the system. ${ }^{11,67}$ Such a model has been often employed in previous studies, ${ }^{9-11,68}$ but it does not give physical insights into the microscopic features of the system. We thus adopted a model that relies on a more physical description of the relaxation process. ${ }^{69}$ We first note that
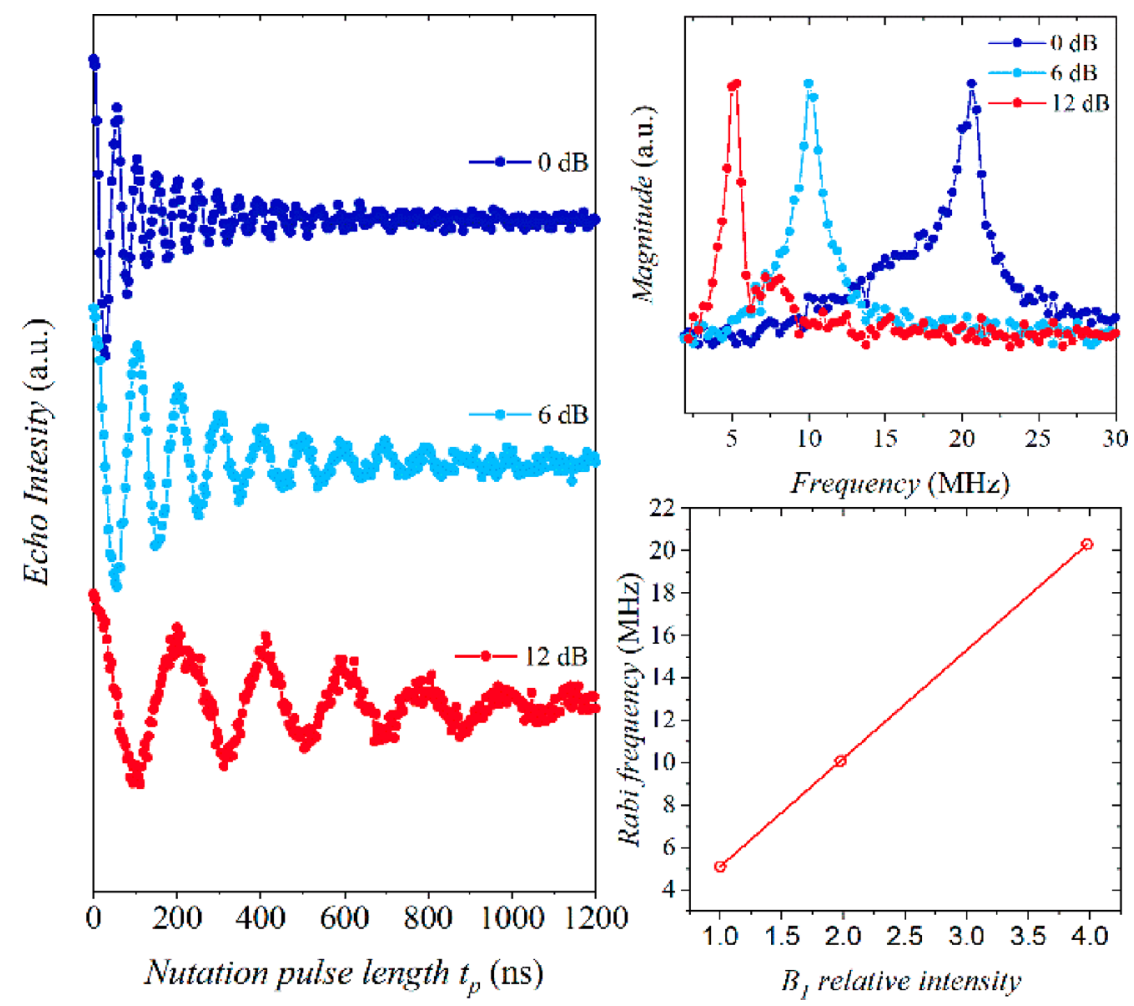

Figure 6. Rabi oscillations observed for $\mathbf{1}_{0.1 \%}$ at $50 \mathrm{~K}$ for three different attenuation values of 0,6 , and $12 \mathrm{~dB}$. On the right side are reported the Fourier transform of the echo intensities (top) and the linear dependence of the Rabi frequencies with respect to the $B_{1}$ relative strength. 
Raman relaxation due to lattice vibrations, namely acoustic phonons, and local-mode relaxation, ${ }^{39}$ generally associated with the activity of optical phonons, are both due to a twophonon spin relaxation mechanism. The usual distinction between the two mechanisms is only practical, and it is made to account for the different $T$ dependences of the relaxation rate. In the case of acoustic modes following a Debye model, Raman relaxation rate is predicted to vary as $T^{9}$, in clear disagreement with experimental findings for $S=1 / 2$ magnetic molecules. ${ }^{10}$ Instead, as it has been recently shown in ref 69, the use of optical phonons as a source of Raman relaxation leads to a relaxation rate proportional to $T^{2}$, in much closer agreement with experimental findings. The latter mechanism is equivalent to a local-mode relaxation mechanism, ${ }^{39}$ and we adopted it to model the experimental data in this work with eq 4. This includes the contribution of the molecular vibrational properties and considers both the energy of vibrational modes and the associated SPC. The model was then rearranged on the basis of several assumptions reported in Pulsed EPR measurement in the Supporting Information).

$$
\begin{aligned}
T_{1}^{-1}= & \tilde{a}_{\text {dir }} \frac{\exp \left(\hbar \omega_{1} / k_{\mathrm{B}} T\right)}{\exp \left(\hbar \omega_{1} / k_{\mathrm{B}} T\right)-1} \\
& +\tilde{a}_{\mathrm{Ram}} \sum_{i} V_{i}^{\mathrm{SP}} \frac{\exp \left(\hbar \omega_{i}^{\mathrm{s}-\mathrm{ph}} / k_{\mathrm{B}} T\right)}{\left(\exp \left(\hbar \omega_{i}^{\mathrm{s}-\mathrm{ph}} / k_{\mathrm{B}} T\right)-1\right)^{2}}
\end{aligned}
$$

The first term resembles the direct contribution to the relaxation in which $\tilde{a}_{\text {dir }}$ is a pre-exponential parameter, and $\omega_{1}$ represents the Zeeman splitting under the operational conditions, which was fixed to the Q-band characteristic energy of about $\sim 1.17 \mathrm{~cm}^{-1}$. The second term is a sum over all of the possible contributions arising from the whole set of normal modes, and it is representative of the Raman contribution to the relaxation. Here, $\omega_{i}^{s-p h}$ represents the energy of the $i$ th optical mode and $V_{i}^{s-p h}$ is the SPC of the employed mode. Furthermore, $\tilde{a}_{\text {Ram }}$ is a parameter which rescales the spin-phonon contribution to the relaxation process. $A b$ initio calculations of normal-mode frequencies and their corresponding SPC properties were performed for compounds $\mathbf{1}$ and $\mathbf{2}$ to minimize the number of fitting parameters.

Ab Initio Spin Dynamics. Atomistic simulation techniques were applied to copper complexes to catch how the structural differences between the molecular systems affect the composition of the normal modes of vibration. Furthermore, their influence on the spin state can be estimated through the SPC coefficients. This approach can lead to a detailed comparison between the SPC of slightly different systems along the whole vibrational spectrum.

The low-energy spectrum of the $S=1 / 2$ systems analyzed here is composed by a Kramers doublet of spin levels, whose degeneracy is removed by the external magnetic field. The total electronic Hamiltonian can be written as $\hat{H}_{0}=\hat{H}_{\mathrm{BO}}+\hat{H}_{\mathrm{SO}}$, where $\hat{H}_{\mathrm{BO}}$ is the nonrelativistic Born-Oppenheimer Hamiltonian and $\hat{H}_{\mathrm{SO}}$ takes into account all spin-orbit interactions. The nuclear degrees of freedom are the $3 N-6$ normal modes of vibrations (phonons), calculated from the Hamiltonian $\hat{H}_{\mathrm{ph}}=\hbar \omega_{a}\left(\hat{n}_{a}+\frac{1}{2}\right)$, where $\hat{n}=\hat{a}^{\dagger} \hat{a}$ is the phonon number operator and $\hat{a}^{\dagger}$ and $\hat{a}$ are the creation and annihilation operators for a phonon of frequency $\omega_{a}$.

Molecular vibrations modulate the spin-orbit interaction. ${ }^{70}$ Indeed, the larger the SPC, e.g. modulation of the $\mathbf{g}$ tensor upon geometrical perturbations through SPC, the shorter the expected $T_{1}$. The Hamiltonian term that describes the firstorder SPC is linear in the ionic displacement, $\hat{q}_{k}=\frac{1}{\sqrt{2}}\left(\hat{a}_{k}^{\dagger}+\hat{a}_{k}\right)$, and can be written as ${ }^{71,72}$

$$
\hat{H}_{\mathrm{s}-\mathrm{ph}}=\sum_{a} V_{a}^{\mathrm{s}-\mathrm{ph}} \hat{q}_{a}=\sum_{a}\left(\frac{\partial \hat{H}_{0}}{\partial q_{a}}\right)_{0} \hat{q}_{a}
$$

The dynamics of the whole system (spin and phonons) follows the equation of the time evolution of the density operator

$$
\frac{\mathrm{d} \hat{\rho}(t)}{\mathrm{d} t}=\frac{i}{\hbar}[\hat{\rho}(t), \hat{H}]
$$

where $\hat{H}=\hat{H}_{0}+\hat{H}_{\mathrm{ph}}+\hat{H}_{\mathrm{s}-\mathrm{ph}}$ is the total Hamiltonian. Equation 6 can be simplified by assuming that the phonon dynamics is much faster than the spin dynamics. The Born-Markov approximation allows tracing the phonons' degrees of freedom of the density matrix. It reduces the problem to a purely electronic one in the presence of a phonon bath. ${ }^{73}$ The dynamics of the spin degrees of freedom can then be studied through the first-order reduced spin density $\hat{\rho}^{\mathrm{S}}$, as described by the diagonal elements of the Redfield equation. ${ }^{74}$

A first qualitative fingerprint of this mechanism, shedding light on the interaction between magnetism and vibrations in each molecule, requires the calculation of the phonon spectrum and the amplitude of spin-phonon coupling (SPC) for all possible modes, $V_{a}^{s-p h}=\left(\frac{\partial \hat{H}_{0}}{\partial q_{a}}\right)$.

The SPC intensities extracted from the calculations are reported in Figure 7. Further details on the calculation of static magnetic properties, normal modes of vibration, and spinphonon coupling are reported in Experimental and Theoretical Details.

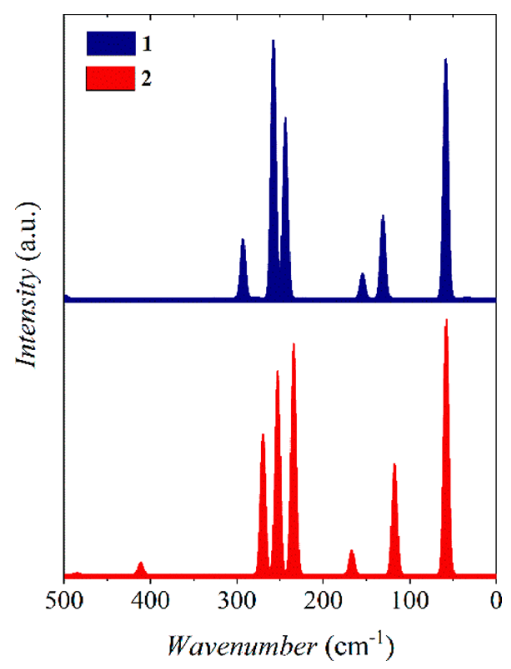

Figure 7. Plot of SPC intensities (in au) as a function of normal mode energy extracted from calculations conducted on $\mathbf{1}$ and $\mathbf{2}$ by adopting the model reported in the main text.

At first glance, a qualitative analysis of chemical differences between $\mathbf{1}$ and $\mathbf{2}$ helps rationalize the results of the calculations. Compound 1 shows eight nitrogen atoms bonding with the eight $\beta$-carbon atoms of pyrrole and bridging four sulfur atoms. Hence, the four $\beta$ substituents form a fivemembered ring that does not increase the strain by acting on 
the peripheral carbon atoms of this tetrakis(thiadiazole)tetraazaporphyrin. The same feature is present in $\mathbf{2}$, and it is strengthened by the phenyl substituents, establishing a higher conjugation along the four isoindole groups of this tetrabenzotetraazaporphyrin. Such outlined features modulate the structural rigidity of 2 , which cannot be considered as flexible as $\mathbf{1}$, considering the first normal mode of vibration occurring at $11.21 \mathrm{~cm}^{-1}$ for 1 and $17.38 \mathrm{~cm}^{-1}$ for 2 (see Tables S3 and S4). ${ }^{41}$ The difference is not so particularly relevant if one considers the coupling of the spin system and the vibrational modes.

The two compounds 1 and 2 belong to the idealized $D_{4 h}$ point group, whose group order is 16 with 10 representations (hereafter "reps" for brevity): $A_{1 g}, A_{2 g}, B_{1 g}, B_{2 g}, E_{g}, A_{1 w}, A_{2 w}$, $\mathrm{B}_{1 \mathrm{u}}, \mathrm{B}_{2 \mathrm{u}}$, and $\mathrm{E}_{\mathrm{u}}{ }^{75}$ The inversion center allows us to discriminate Raman-active modes (gerade) from IR-active modes (ungerade). One sample normal mode is shown for each symmetry in Figure S26 (1) and Figure S27 (2). The most effective normal modes, i.e., those bearing the highest couplings with the spin systems, are found to show the same reps of d orbitals. ${ }^{76}$ The active modes showing the highest SPC constants over an arbitrary threshold of $10^{-7}$ and for $\omega<500$ $\mathrm{cm}^{-1}$ are reported for compounds $\mathbf{1}$ and $\mathbf{2}$ in Table 2. The complete list of modes with their relative SPC constants is instead available in Table S3 (1) and Table S4 (2).

Table 2. Calculated Values of $\omega\left(\mathrm{cm}^{-1}\right)$ and SPC (au) for 1 and 2 at $\omega<500 \mathrm{~cm}^{-1}$ and SPC $>10^{-7}$ Reported with Their Respective Representations Expressed as Mulliken Notations

\begin{tabular}{|c|c|c|c|c|c|}
\hline \multicolumn{3}{|c|}{1} & \multicolumn{3}{|c|}{2} \\
\hline$\omega\left(\mathrm{cm}^{-1}\right)$ & SPC (au) & Reps. & $\omega\left(\mathrm{cm}^{-1}\right)$ & SPC (au) & $\overline{\text { Reps. }}$ \\
\hline 58.161 & $1.77 \times 10^{-5}$ & $\mathrm{E}_{\mathrm{g}}$ & 57.582 & $1.48 \times 10^{-5}$ & $\mathrm{E}_{\mathrm{g}}$ \\
\hline 58.471 & $1.61 \times 10^{-5}$ & $\mathrm{E}_{\mathrm{g}}$ & 58.048 & $1.45 \times 10^{-5}$ & $\mathrm{E}_{\mathrm{g}}$ \\
\hline 131.03 & $5.82 \times 10^{-6}$ & $\mathrm{E}_{\mathrm{g}}$ & 117.59 & $6.41 \times 10^{-6}$ & $\mathrm{E}_{\mathrm{g}}$ \\
\hline 131.27 & $5.85 \times 10^{-6}$ & $\mathrm{E}_{\mathrm{g}}$ & 117.77 & $6.32 \times 10^{-6}$ & $\mathrm{E}_{\mathrm{g}}$ \\
\hline 154.79 & $3.48 \times 10^{-6}$ & $B_{1 g}$ & 167.31 & $2.79 \times 10^{-6}$ & $B_{1 g}$ \\
\hline 244.19 & $2.55 \times 10^{-5}$ & $A_{1 g}$ & 234.47 & $1.32 \times 10^{-5}$ & $\mathrm{E}_{\mathrm{g}}$ \\
\hline 257.81 & $1.87 \times 10^{-5}$ & $E_{g}{ }^{\circ}$ & 234.55 & $1.32 \times 10^{-5}$ & $A_{1 g}^{\circ}$ \\
\hline 257.92 & $1.77 \times 10^{-5}$ & $E_{g}$ & 253.06 & $2.33 \times 10^{-5}$ & $E_{g}$ \\
\hline 277.85 & $1.63 \times 10^{-7}$ & $\mathrm{~B}_{2 \mathrm{~g}}$ & 270.02 & $8.06 \times 10^{-6}$ & $\mathrm{E}_{\mathrm{g}}$ \\
\hline 293.09 & $4.08 \times 10^{-6}$ & $\mathrm{E}_{\mathrm{g}}$ & 270.09 & $8.02 \times 10^{-6}$ & $\mathrm{E}_{\mathrm{g}}$ \\
\hline 293.21 & $4.26 \times 10^{-6}$ & $E_{g}$ & 411.51 & $6.91 \times 10^{-7}$ & $E_{g}$ \\
\hline 499.08 & $2.18 \times 10^{-7}$ & $E_{g}^{b}$ & 411.59 & $6.96 \times 10^{-7}$ & $B_{2 g}^{8}$ \\
\hline 499.20 & $1.85 \times 10^{-7}$ & $\mathrm{E}_{\mathrm{g}}$ & & & \\
\hline
\end{tabular}

The active vibrations displace the donor atoms out of the plane of the porphyrazine core, as visible in $\mathrm{E}_{\mathrm{g}}$ modes 10 and 11 of 1 (Figure S26) and $E_{g}$ modes 10 and 11 of 2 (Figure S27), or in-plane, back and forth along with the $\mathrm{Cu}-\mathrm{N}$ directions $\left(\mathrm{A}_{1 \mathrm{~g}}, \mathrm{~B}_{1 \mathrm{~g}}\right.$, and $\mathrm{B}_{2 \mathrm{~g}}$ modes 23,20 , and 21 of $\mathbf{1}$ and 26 , 20 , and 23 of 2 ) (Figure $S 26$ ). The ungerade modes that cause out-of-plane displacements $\left(A_{1 u}, A_{2 u}, B_{1 w}\right.$ and $B_{2 u}$ modes 39, 19, 22, and 18) (Tables $S 3$ and $S 4$ ) have low couplings since they leave the four donor atoms still or displace them rigidly. A rigid shift of the metal atom and its first coordination sphere is also given by the in-plane modes corresponding to rotations $\left(A_{2 g}\right.$ mode 22 of 1 and 21 of 2$)$ and translations ( $E_{u}$ modes 13 and 14 of 1 and 16 and 17 of 2) (Table S3 and S4), and therefore, they show small SPC. In summary, the first coordination sphere is strongly perturbed only by gerade modes.

\section{DISCUSSION}

In the present study, we observed that removing active nuclei from peripheral positions of $\mathbf{1}$ entails a moderate but appreciable coherence gain with respect to compound 2, when the electronic spin dilution reduces the electron spinspin contribution. We notice that $T_{\mathrm{m}}$ values significantly longer than those measured here were reported for thin films ${ }^{8}$ of 2 and for frozen solution in $\mathrm{D}_{2} \mathrm{SO}_{4} \cdot{ }^{77}$ We therefore tried to further validate such a nuclear spin economy strategy by comparing the behavior of the two complexes in diluted solutions of $\mathrm{D}_{2} \mathrm{SO}_{4}$, but the porphyrazine complex turned out to show significantly modified EDFS spectra under these conditions, as evidenced in Figure S24.

In the analysis of $T_{1}$, the information gained through the $a b$ initio investigation of the spin and vibrational properties of 1 and 2 allows us to rationalize the spin dynamics of the two molecules. In the model described by eq 4 , the whole set of vibrational energies and spin-phonon coupling coefficients resulting from $a b$ initio calculations are adopted to define $\omega_{i}^{s-p h}$ and $V_{i}^{\text {s-ph }}$, respectively. It follows that only two parameters of the model are left free to vary. The first is $\tilde{a}_{\text {dir }}$ its independent computation will require the computation of acoustic phonons out of the $\Gamma$ point of the Brillouin space where energy matching with Zeeman splitting is observed. This goes far beyond the scope of our isolated molecule calculation. Additionally, the unique scale factor $\tilde{a}_{\text {Ram }}$ connecting the SPC to the relaxation rate is introduced for the whole set of vibrational modes to preserve the relative weight of each mode in the relaxation process. Best-fit parameters are given in Table 3.

Table 3. Best-Fit Parameters Obtained Using the Model (4) to Reproduce the Temperature Dependence of $T_{1}$ Shown in Figure 5

\begin{tabular}{ccc} 
& $\tilde{a}_{\text {dir }}\left(\mathrm{m} \mathrm{s}^{-1}\right)$ & $\tilde{a}_{\text {Ram }}\left(\mathrm{ms}^{-1}\right)$ \\
$\mathbf{1}_{\mathbf{0 . 1} \%}$ & $(3.32 \pm 1.47) \times 10^{-2}$ & $(1.99 \pm 0.41) \times 10^{6}$ \\
$\mathbf{2}_{0.1 \%}$ & $(3.87 \pm 0.64) \times 10^{-1}$ & $(4.20 \pm 0.55) \times 10^{6}$ \\
\hline
\end{tabular}

An extensive comparison between the spin-lattice relaxation processes of the two systems is provided by the multitechnique approach employed. As shown in Figure 5, the $T_{1}$ trends of $\mathbf{1}_{\mathbf{0 . 1} \%}$ and $\mathbf{2}_{\mathbf{0 . 1} \%}$ above $40 \mathrm{~K}$ are similar, and they are comparable with AC susceptibility data of $\mathbf{1}_{\mathbf{2 0} \%}$ and $\mathbf{2}_{\mathbf{2 0} \%}$ (Figure 3 ). This is also highlighted by the results of the fits reported in Table 3. Indeed, a similar weight of Raman contribution emerged from the fit performed with the alternative employed model (4). The further analysis of the single-mode contribution to the relaxation rate indicated that the most effective modes are those with SPC coefficients higher than $10^{-7}$ and energies lower than $500 \mathrm{~cm}^{-1}$. Among them, the first two gerade optical modes placed at 58.16 and $58.47 \mathrm{~cm}^{-1}\left(\mathbf{1}_{0.1 \%}\right)$ and 57.78 and $58.05 \mathrm{~cm}^{-1}\left(2_{0.1 \%}\right)$ have the most influence on the relaxation (Figure S23). Higher energy optical modes or modes with lower SPC introduce a small contribution to the relaxation of the two compounds. In order to understand why relatively high energy phonons, such as the first optical phonons, can contribute to spin relaxation, it must be stressed that the thermal population is not the only ingredient determining the spin relaxation rate. Indeed, the vibrational density of states and the magnitude of SPC are two additional quantities that need to be carefully considered. Both of these quantities are 
much larger for optical phonons than for acoustic phonons and largely compensate for the difference in the thermal population.

The striking dependence of the SPC on the symmetry of the involved vibrational modes is remarkable. Strong SP coupling characterizes gerade modes, of which $\mathrm{A}_{1 \mathrm{~g}}$ modes present the most intense coupling constant in both compounds (Table 2). This can be explained by considering that, in copper(II) systems, the orbital contribution to the $\mathbf{g}$ tensor comes from the admixing of the ground state with excited states through spin-orbit coupling. For the analyzed systems, the unpaired electron occupies the $\mathrm{d}_{x^{2}-y^{2}}$ orbital, which points toward the $\mathrm{Cu}-\mathrm{N}$ bonds. The distortion carried out by symmetric vibrations modifies the overlap between metal and ligand orbitals, significantly affecting the energy difference between the ground and excited states. Consequently, this modifies the weight of the spin-orbit-induced perturbation. In contrast, asymmetric vibrations present an almost null net variation of the overlap (see Figure 8).
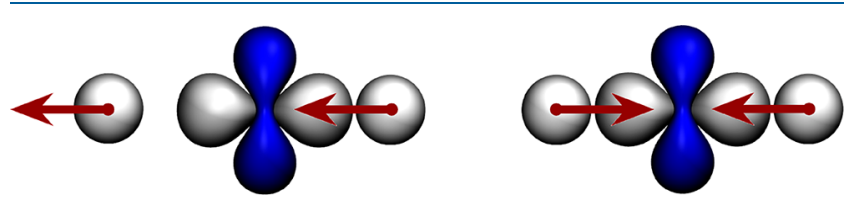

Figure 8. Schematic representation of the overlap variation between the two nitrogen $\sigma$ orbitals interacting with the copper $\mathrm{d}_{x^{2}-y^{2}}$ orbital in response to asymmetric (left) and symmetric (right) displacements in molecular vibrations.

Disentangling the role of different types of vibrations is mandatory for a rational design of molecules exhibiting long coherence times over a wide temperature range.

On the other hand, below $40 \mathrm{~K} T_{1}$ trends are divergent for the two compounds, and this is reflected in the different values of the fitting parameter $\tilde{a}_{\text {dir }}$. By relation of this evidence with what has been extracted from AC susceptibility measurements, it emerges that, at high concentration, the direct mechanism of relaxation is affected by spin-spin interactions, which are similar for the two compounds having comparable $\mathrm{Cu}-\mathrm{Cu}$ distances. When the spin concentration is lowered to $0.1 \%$, it appears that the spin-lattice relaxation is less efficient for the porphyrazine complex than for the phthalocyanine complex. We hypothesize that this might be related to the role of the highly polarizable sulfur atoms, which can be relevant for van der Waals interactions affecting the acoustic phonon dispersion, but further calculations are necessary to confirm this hypothesis.

\section{CONCLUSIONS}

In conclusion, our investigations conducted with pulsed EPR spectroscopy verified that both $T_{1}$ and $T_{\mathrm{m}}$ follow different temperature dependences when the magnetic dilution is pushed below the $1 \%$ limit. We proved experimentally that a coherence time enhancement of porphyrazine-based complexes might be achieved by replacing peripheral hydrogen atoms of $[\mathrm{Cu}(\mathrm{Pc})]$ with thiadiazole units of $[\mathrm{Cu}(\mathrm{TTDPz})]$, partially highlighting that hydrogen atoms placed around the 6 $\AA$ spin diffusion barrier affect the coherence time of the central ion. ${ }^{38,40}$ On the other hand, the temperature dependence of $T_{1}$ was rationalized by $a b$ initio calculations, allowing us to gain microscopic insights into spin-lattice relaxation mechanisms.
In the limit of the assumptions mentioned above, the model adopted to fit the temperature dependence of $T_{1}$ data ${ }^{69}$ revealed that the major difference between the two compounds lies in the efficiency of the direct mechanism dominating the low-temperature regime. In contrast, the contributions of spin-phonon coupling to the Raman relaxation mechanism have similar weights in the two compounds. This is confirmed by $a b$ initio calculations, evidencing the pivotal role played by the low-energy vibrational modes at around $57 \mathrm{~cm}^{-1}$ for both compounds. Furthermore, vibrational modes with gerade symmetry are the most coupled modes, confirming previous indications of the crucial role played by the symmetry of vibrations. ${ }^{71}$ In such cases, Raman spectroscopy in the terahertz regime, more demanding than absorption measurements, appears to be necessary for a complete experimental characterization of the vibrational properties of relevance for the spin dynamics of centrosymmetric $3 \mathrm{~d}$-metal complexes.

\section{EXPERIMENTAL AND THEORETICAL DETAILS}

General Remarks. All chemicals were purchased and used without further purification. The $\mathrm{Mg}$ metal used for the preparation of $\mathrm{Mg}(\mathrm{PrO})_{2}$ was previously treated with a dilute solution of $\mathrm{HCl}$ to remove impurities, stabilized in acetone, and dried under vacuum over $\mathrm{P}_{2} \mathrm{O}_{5}$ overnight. All glassware was previously dried at $120{ }^{\circ} \mathrm{C}$ overnight. The reaction was conducted using anhydrous propanol 99\% purchased from Alfa Aesar. A classic Schlenk technique was adopted using dried $\mathrm{N}_{2}$ as the inert gas.

All compounds were obtained according to previously reported procedures. ${ }^{45,46,54}$ All of the employed synthetic procedures are reported in detail in the Supporting Information. Diamagnetic dilutions $\mathbf{1}_{20 \%}, \mathbf{2}_{20 \%}, \mathbf{1}_{2 \%}, \mathbf{1}_{0.1 \%}$, and $\mathbf{2}_{0.1 \%}$ were obtained as follows: stoichiometric amounts of the paramagnetic species and its diamagnetic analogue (total amount $100 \mathrm{mg}$ ) were added together to methanesulfonic acid $(30 \mathrm{~mL})$ and stirred for $15 \mathrm{~min}$. After the complete dissolution of compounds, cold 2-propanol $(40 \mathrm{~mL})$ was added dropwise with stirring to the solution cooled at $0{ }^{\circ} \mathrm{C}$. Centrifugation and subsequent washing cycles with water and acetone led to the isolation of blue amorphous solids of $\mathbf{1}_{20 \%}, \mathbf{1}_{2 \%}$, and $\mathbf{1}_{\mathbf{0 . 1}}$. Compounds $\mathbf{2}_{\mathbf{2 0} \%}$ and $\mathbf{2}_{0.1 \%}$ were instead filtered while being kept cold and stored at $-35{ }^{\circ} \mathrm{C}$ to prevent a phase transition to the $\beta$ polymorph.

Powder X-ray Crystallography. Powder X-ray diffraction (PXRD) patterns on polycrystalline samples of $\mathbf{1},[\mathrm{Ni}(\mathrm{TTDP})$ ], $\mathbf{2}_{20 \%}$, and $\mathbf{2}_{0.1 \%}$ were recorded on a Bruker New D8 Advance DAVINCI diffractometer in a $\theta-\theta$ configuration equipped with a linear detector. The patterns were collected within the range $5-50^{\circ}$ $(2 \theta)$ using $\mathrm{Cu} \mathrm{K} \alpha$ radiation $(\lambda=1.5398 \AA)$ with a step of $0.025^{\circ}$ and an exposure time varying from 0.6 to $1 \mathrm{~s} / \mathrm{deg}$. Simulated patterns were generated from the atomic coordinates of the single-crystal structure solutions $^{51,52}$ (CCDC files: 236691, $\alpha$-NiTTDPz; ${ }^{51}$ 236692, $\gamma$ NiTTDPz; ${ }^{51}$ 236689, $\gamma$-CuTTDPz; ${ }^{51} 112723, \mathrm{CuPc}^{52}$ ) using the Mercury CSD 4.0 software $^{78}$ (copyright CCDC, http://www.ccdc. cam.ac.uk/mercury/) using a fwhm (full width at half-maximum) of $0.3^{\circ}$ for $1,0.1^{\circ}$ for $[\mathrm{Ni}(\mathrm{TTDPz})]$, and $0.2^{\circ}$ for 2 , and a $2 \theta$ step of $0.025^{\circ}$.

Magnetic Measurements. Alternating Current (AC) magnetic susceptibility measurements were performed using a Quantum Design Physical Property Measurement System (PPMS). AC susceptibility measurements were achieved in the frequency $(\nu)$ range 10-10000 $\mathrm{Hz}$ by applying an oscillating field of $1 \mathrm{mT}$ in the temperature range $2.0-50 \mathrm{~K}$ with applied static magnetic fields up to $8.5 \mathrm{~T}$. Measurements were performed on randomly oriented powder samples of $[\mathrm{Cu}(\mathrm{TTDPz})](34.6 \mathrm{mg}),[\mathrm{Cu}(\mathrm{Pc})](28.3 \mathrm{mg})$, $\left[\mathrm{Cu}_{0.2} \mathrm{Ni}_{0.8}(\mathrm{TTDPz})\right](47.5 \mathrm{mg})$, and $\left[\mathrm{Cu}_{0.2}(\mathrm{Pc})\right](61.6 \mathrm{mg})$. Susceptibility data were corrected for the sample holder previously measured using the same conditions and for the diamagnetic contributions as deduced by using Pascal's constant tables. 
Electron Paramagnetic Resonance. CW X-band EPR spectra of all samples were recorded on a Bruker Elexsys E500 spectrometer equipped with an SHQ cavity $(v \approx 9.87 \mathrm{GHz}$ for room-temperature setup, $v \approx 9.40 \mathrm{GHz}$ for low-temperature setup). Low-temperature measurements were obtained using an Oxford Instruments ESR900 continuous flow helium cryostat and temperature-controlled by an Oxford Instrument ITC. Pulsed EPR measurements were carried out with a Bruker Elexsys E580 spectrometer at X-band $(\nu=9.75 \mathrm{GHz})$ and Q-band $(\nu=33.78 \mathrm{GHz})$ frequencies equipped with a flexline dielectric ring ENDOR resonator (Bruker EN 4118X-MD4). Temperatures between 4.5 and $250 \mathrm{~K}$ were obtained with an Oxford Instruments CF935 continuous-flow helium cryostat. Echo detected field swept EPR spectra were recorded by using the Hahn Echo pulse sequence $(\pi / 2-t-\pi-t-$ echo $)$ with fixed interpulse delay times of $t=$ $200 \mathrm{~ns}, t_{\pi / 2}=16 \mathrm{~ns}$, and $t_{\pi}=32 \mathrm{~ns}$. Phase memory times were measured by the Hahn echo sequence upon increasing the interpulse delay $\tau$ starting from $\tau=110 \mathrm{~ns}$. Typical pulse lengths were $t_{\pi / 2}=40$ ns and $t_{\pi}=80 \mathrm{~ns}$. Spin-lattice relaxation times were measured using the standard inversion recovery sequence $\left(\pi-t_{\mathrm{d}}-\pi / 2-t-\pi-t-\mathrm{echo}\right)$, with $\pi / 2=16$ ns. The uncertainty in $T_{1}$ estimated from replicate measurements was $5-10 \%$ depending upon the signal to noise ratio at a given temperature-field combination. Nutation measurements were performed with a nutation pulse $\left(t_{\mathrm{p}}\right)$ of variable length followed by a Hahn echo sequence $\left(t_{\mathrm{p}}-t_{\mathrm{d}}-\pi / 2-t-\pi-\mathrm{t}-\mathrm{echo}\right)$ with $t_{\mathrm{d}} \gg T_{\mathrm{m}}$ (i.e. $t_{\mathrm{d}}$ $=5 \mu \mathrm{s}$ ). The interpulse delay $t$ was $200 \mathrm{~ns}$, and the pulse length of the detection sequence was adjusted depending on the attenuation level of $B_{1}$.

Spin Hamiltonian Calculations. Calculations of the spin Hamiltonian parameters were carried out with the ORCA package. ${ }^{79}$ The level of theory used was DFT, with the PBE0 hybrid functional and all-electron basis sets: def2-TZVP basis set for the V, O, and S atoms and def2-SVP for the $\mathrm{C}$ and $\mathrm{H}$ atoms, for the Lande factor, while hyperfine values were calculated by adopting a specialized basis set for accurate hyperfine coupling constants (aug-cc-pVTZ-J). The molecular geometry used for these simulations was obtained by the periodic DFT calculations for the optimized molecular structure in $\mathrm{CP} 2 \mathrm{~K}^{80}$ The more accurate hybrid DFT functional PBE0 contains an exact-exchange contribution lacking in GGA functionals. Thus, the significant potentials imposed by these functionals is different. Hartree-Fock/DFT hybrid functionals admix a certain amount of Fock exact exchange $\left(E_{\mathrm{x}}\right)$ to the exchange part $\left(E_{\mathrm{x}}^{\mathrm{PBE}}\right)$ and correlation part $\left(E_{\mathrm{c}}^{\mathrm{PBE}}\right)$ of the PBE GGA density functional:

$$
E_{\mathrm{xc}}^{\mathrm{PBE} 0}=\frac{1}{4} E_{\mathrm{x}}+\frac{3}{4} E_{\mathrm{x}}^{\mathrm{PBE}}+E_{\mathrm{c}}^{\mathrm{PBE}}
$$

This kind of functional turns out to be more accurate than common local or semilocal DFT functionals.

Normal Mode Calculations. Unrestricted open shell density functional theory based calculations have been performed for the magnetic complexes mentioned above. Strict convergence parameters for DFT structural optimizations are required to improve the agreement between experimental and calculated vibrational spectra (Figure S25). The computational box contains one isolated molecule, allowing calculation of $3 N-6$ localized optical normal mode energies. In the harmonic approximation, the Taylor expansion of potential energy is truncated at second order around the equilibrium nuclei positions, where its first derivatives are supposed to vanish. ${ }^{81}$

The search of a local minimum of the energy, corresponding to the equilibrium geometry of the $N$ atom system, requires a strict minimization procedure to ensure the average total forces are around $1 \times 10^{-8}$ au. The second derivatives of the energy are calculated numerically by finite differences perturbing the equilibrium geometry along the $3 \mathrm{~N}$ coordinates.

The CP2K simulation package provides the algorithm for periodic $a b$ initio calculations of energies and forces. The force constant calculation and diagonalization are implemented within an in-house software; the solution of the eigenproblem gives eigenvalues and eigenvectors representing the atomic weights and energies of $3 \mathrm{~N}$ independent normal modes of vibrations.
The DFT scheme adopted, as implemented in the Quickstep module, ${ }^{82}$ uses pseudopotentials (GTH-PBE) in order to integrate out the core electrons from the problem. The valence pseudowave functions are expanded in Gaussian-type orbitals (DZVP-MOLOPTSR-GTH), and the density is represented in a plane wave auxiliary basis.

The use of a plane wave basis for the electron density permits efficient calculation of the Hartree energy using fast Fourier transforms. The functional approximation by Perdew-BurkeErnzerhof (PBE) $)^{83,84}$ was chosen for the present calculations. However, the basis set size depends on the volume of the cutoff of plane wave energy and the computational box. The basis set functions are discretized on numerical grids in the real space. Therefore, adding high-frequency plane waves will require increasingly finer grid points to be described, which will quickly increase the computational cost. It was ascertained that a cutoff of at least $1000 \mathrm{Ry}$ was necessary to achieve a good quality of vibrational spectra in the low-energy range $\left(0-100 \mathrm{~cm}^{-1}\right)$.

The symmetry properties of a normal mode with wave vector $\mathbf{k}$ can be determined by studying the transformation properties of the creation and annihilation operators $a^{+}$and $a$ under the operation of the point group of the molecule and assigning them to the appropriate representation. $^{85}$

Spin-Phonon Coupling Calculations. The method to investigate the spin-phonon coupling has been developed in previous works ${ }^{72}$ and is based on the calculation of spin-phonon coupling within a perturbative approach. The unperturbed Hamiltonian $H_{0}$ is evaluated by distorting the equilibrium structure in six points along each normal mode; fitting the $\frac{\partial \hat{H}_{0}}{\partial q_{a}}$ vs $q_{a}$ displacement with a secondorder polynomial and taking the first-order coefficient, we obtain directly the first derivative calculated numerically by finite differences.

The $H_{0}$ value for an $S=\frac{1}{2}$ spin system contains only the Zeeman term $\hat{H}_{\text {Zeeman }}=\mu_{\mathrm{B}} \mathbf{B} \cdot \boldsymbol{g} \cdot \hat{S}$, where the only parameter depending on nuclear coordinates is the Landé factor $g$. The interaction Hamiltonian hence assumes the form

$$
\hat{H}_{s-\mathrm{ph}}=\sum_{a}\left(\frac{\partial \hat{H}_{0}}{\partial q_{a}}\right)_{0} \hat{q}_{a}=\sum_{a} \sum_{j, r} \mu_{\mathrm{B}} B_{j} \hat{S}_{r}\left(\frac{\partial g_{j r}}{\partial q_{a}}\right)_{0} \hat{q}_{a}
$$

Calculation of the spin-phonon Hamiltonian parameters was carried out with the ORCA package. The level of theory used was DFT, with the PBE0 hybrid functional as for static magnetic property calculations. A balanced polarized triple- $\zeta$ basis set was adopted (def2-TZVP) for metal and sulfur atoms, while a polarized double- $\zeta$ basis set (def2-SVP) was used for light elements such as carbon and hydrogen atoms. The molecular geometry employed for these simulations was obtained by the periodic DFT calculation of the optimized molecular structure in CP2K.

\section{ASSOCIATED CONTENT}

\section{Supporting Information}

The Supporting Information is available free of charge at https://pubs.acs.org/doi/10.1021/acs.inorgchem.0c02573.

Additional figures and data, as mentioned in the text (PDF)

\section{AUTHOR INFORMATION}

\section{Corresponding Authors}

Enrico Salvadori - Dipartimento di Chimica e NIS Centre, Università di Torino, I10125 Torino, Italy; 이이.org/ 0000-0003-4394-9438; Email: enrico.salvadori@unito.it

Roberta Sessoli - Dipartimento di Chimica "Ugo Schiff" \& INSTM RU, Università degli Studi di Firenze, I50019 Firenze, Italy; $\odot$ orcid.org/0000-0003-3783-2700; Email: roberta.sessoli@unifi.it 


\section{Authors}

Fabio Santanni - Dipartimento di Chimica "Ugo Schiff" \& INSTM RU, Università degli Studi di Firenze, I50019 Firenze, Italy; ○ orcid.org/0000-0002-0506-8333

Andrea Albino - Dipartimento di Chimica "Ugo Schiff" \& INSTM RU, Università degli Studi di Firenze, I50019 Firenze, Italy

Matteo Atzori - Laboratoire National des Champs Magnétiques Intenses (LNCMI), Univ. Grenoble Alpes, INSA Toulouse, Univ. Toulouse Paul Sabatier, EMFL, CNRS, F38043 Grenoble, France; 이이. ord.0000-0003-13576159

Davide Ranieri - Dipartimento di Chimica "Ugo Schiff" \&" INSTM RU, Università degli Studi di Firenze, I50019 Firenze, Italy

Mario Chiesa - Dipartimento di Chimica e NIS Centre, Università di Torino, I10125 Torino, Italy; ○orcid.org/ 0000-0001-8128-8031

Alessandro Lunghi - School of Physics, AMBER and CRANN Institute, Trinity College, Dublin 2, Ireland; () orcid.org/0000-0002-1948-4434

Andrea Bencini - Dipartimento di Chimica "Ugo Schiff" \& INSTM RU, Università degli Studi di Firenze, I50019 Firenze, Italy

Lorenzo Sorace - Dipartimento di Chimica "Ugo Schiff" \& INSTM RU, Università degli Studi di Firenze, I50019 Firenze, Italy; 이이이.org/0000-0003-4785-1331

Federico Totti - Dipartimento di Chimica "Ugo Schiff" \& INSTM RU, Università degli Studi di Firenze, I50019

Firenze, Italy; ○) orcid.org/0000-0003-4752-0495

Complete contact information is available at:

https://pubs.acs.org/10.1021/acs.inorgchem.0c02573

\section{Author Contributions}

${ }^{\perp}$ F.S. and A.A. contributed equally to this work.

\section{Notes}

The authors declare no competing financial interest.

\section{ACKNOWLEDGMENTS}

Financial support by the EU Commission through the QuantERA Project SUMO, the FETOPEN project FATMOLS (GA 862893), and the MOLSPIN COST action CA15128 project is acknowledged. The support by the Italian MIUR through PRIN 2015 (Project HYFSRT) and Progetto Dipartimenti di Eccellenza 2018-2022 (ref no. B96C1700020008), by the Fondazione Cassa di Risparmio di Firenze, and by AMBER (grant/12/RC/2278 P2) is also acknowledged. The computing resources and the related technical support used for this work have been provided by CRESCO/ENEAGRID High Performance Computing infrastructure and its staff ${ }^{86}$ funded by ENEA, the Italian National Agency for New Technologies, Energy and Sustainable Economic Development, and by Italian and European research programs; see http://www.cresco.enea.it/english for information.

\section{REFERENCES}

(1) Nielsen, M. A.; Chuang, I. L. Quantum Computation and Quantum Information; Cambridge University Press: Cambridge, 2000.

(2) Harrow, A. W.; Montanaro, A. Quantum Computational Supremacy. Nature 2017, 549, 203-209.

(3) Arute, F.; Arya, K.; Babbush, R.; Bacon, D.; Bardin, J. C.; Barends, R.; Biswas, R.; Boixo, S.; Brandao, F. G. S. L.; Buell, D. A.; et al. Quantum Supremacy Using a Programmable Superconducting Processor. Nature 2019, 574, 505-509.

(4) Deutsch, D. Quantum Theory, the Church-Turing Principle and the Universal Quantum Computer. Proc. R. Soc. A Math. Phys. Eng. Sci. 1985, 400, 97-117.

(5) Bermudez, A.; Xu, X.; Nigmatullin, R.; Gorman, J. O.; Negnevitsky, V.; Schindler, P.; Monz, T.; Poschinger, U. G.; Hempel, C.; Home, J. Assessing the Progress of Trapped-Ion Processors Towards Fault-Tolerant Quantum Computation. Phys. Rev. X 2017, 7, 041061.

(6) Barends, R.; Kelly, J.; Megrant, A.; Veitia, A.; Sank, D.; Jeffrey, E.; White, T. C.; Mutus, J.; Fowler, A. G.; Campbell, B.; et al. Superconducting Quantum Circuits at the Surface Code Threshold for Fault Tolerance. Nature 2014, 508, 500-503.

(7) Moll, N.; Barkoutsos, P.; Bishop, L. S.; Chow, J. M.; Cross, A.; Egger, D. J.; Filipp, S.; Fuhrer, A.; Gambetta, J. M.; Ganzhorn, M.; et al. Quantum Optimization Using Variational Algorithms on NearTerm Quantum Devices. Quantum Sci. Technol. 2018, 3, 030503.

(8) Warner, M.; Din, S.; Tupitsyn, I. S.; Morley, G. W.; Stoneham, A. M.; Gardener, J. A.; Wu, Z.; Fisher, A. J.; Heutz, S.; Kay, C. W. M.; et al. Potential for Spin-Based Information Processing in a Thin-Film Molecular Semiconductor. Nature 2013, 503, 504-508.

(9) Atzori, M.; Tesi, L.; Morra, E.; Chiesa, M.; Sorace, L.; Sessoli, R. Room-Temperature Quantum Coherence and Rabi Oscillations in Vanadyl Phthalocyanine: Toward Multifunctional Molecular Spin Qubits. J. Am. Chem. Soc. 2016, 138, 2154-2157.

(10) Atzori, M.; Morra, E.; Tesi, L.; Albino, A.; Chiesa, M.; Sorace, L.; Sessoli, R. Quantum Coherence Times Enhancement in Vanadium(IV)-Based Potential Molecular Qubits: The Key Role of the Vanadyl Moiety. J. Am. Chem. Soc. 2016, 138, 11234-11244.

(11) Yamabayashi, T.; Atzori, M.; Tesi, L.; Cosquer, G.; Santanni, F.; Boulon, M.; Morra, E.; Benci, S.; Torre, R.; Chiesa, M.; et al. Scaling Up Electronic Spin Qubits into a Three-Dimensional MetalOrganic Framework. J. Am. Chem. Soc. 2018, 140, 12090-12101.

(12) Bader, K.; Dengler, D.; Lenz, S.; Endeward, B.; Jiang Da, S.; Neugebauer, P.; Van Slageren, J. Room Temperature Quantum Coherence in a Potential Molecular Qubit. Nat. Commun. 2014, 5, 15.

(13) Borilovic, I.; Alonso, P. J.; Roubeau, O.; Aromì, G. A BisVanadyl Coordination Complex as a 2-Qubit Quantum Gate. Chem. Commun. 2020, 56, 3139-3142.

(14) Atzori, M.; Chiesa, A.; Morra, E.; Chiesa, M.; Sorace, L.; Carretta, S.; Sessoli, R. A Two-Qubit Molecular Architecture for Electron-Mediated Nuclear Quantum Simulation. Chem. Sci. 2018, 9, 6183-6192.

(15) Clarke, J.; Wilhelm, F. K. Superconducting Quantum Bits. Nature 2008, 453, 1031-1042.

(16) Devoret, M. H.; Schoelkopf, R. J. Superconducting Circuits for Quantum Information: An Outlook. Science 2013, 339, 1169-1174.

(17) Friis, N.; Marty, O.; Maier, C.; Hempel, C.; Holzäpfel, M.; Jurcevic, P.; Plenio, M. B.; Huber, M.; Roos, C.; Blatt, R.; et al. Observation of Entangled States of a Fully Controlled 20-Qubit System. Phys. Rev. X 2018, 8, 21012.

(18) Fuchs, G. D.; Burkard, G.; Klimov, P. V.; Awschalom, D. D. A Quantum Memory Intrinsic to Single Nitrogen-Vancancy Centres in Diamond. Nat. Phys. 2011, 7, 789-793.

(19) Petit, L.; Eenink, H. G. J.; Russ, M.; Lawrie, W. I. L.; Hendrickx, N. W.; Philips, S. G. J.; Clarke, J. S.; Vandersypen, L. M. K.; Veldhorst, M. Universal Quantum Logic in Hot Silicon Qubits. Nature 2020, 580, 355-359.

(20) Vandersypen, L. M. K.; Bluhm, H.; Clarke, J. S.; Dzurak, A. S.; Ishihara, R.; Morello, A.; Reilly, D. J.; Schreiber, L. R.; Veldhorst, M. Interfacing Spin Qubits in Quantum Dots and Donors-Hot, Dense, and Coherent. npj Quantum Inf. 2017, 3, 34.

(21) Zwanenburg, F. A.; Dzurak, A. S.; Morello, A.; Simmons, M. Y.; Hollenberg, L. C. L.; Klimeck, G.; Rogge, S.; Coppersmith, S. N.; Eriksson, M. A. Silicon Quantum Electronics. Rev. Mod. Phys. 2013, 85, 961-1019. 
(22) Koch, M.; Keizer, J. G.; Pakkiam, P.; Keith, D.; House, M. G.; Peretz, E.; Simmons, M. Y. Spin Read-out in Atomic Qubits in an AllEpitaxial Three-Dimensional Transistor. Nat. Nanotechnol. 2019, 14, 137-140.

(23) He, Y.; Gorman, S. K.; Keith, D.; Kranz, L.; Keizer, J. G.; Simmons, M. Y. A Two-Qubit Gate between Phosphorus Donor Electrons in Silicon. Nature 2019, 571, 371-375.

(24) DiVincenzo, D. P. The Physical Implementation of Quantum Computing. Fortschr. Phys. 2000, 48, 771.

(25) Atzori, M.; Sessoli, R. The Second Quantum Revolution: Role and Challenges of Molecular Chemistry. J. Am. Chem. Soc. 2019, 141, 11339-11352.

(26) Wasielewski, M. R.; Forbes, M. D. E.; Frank, N. L.; Kowalski, K.; Scholes, G. D.; Yuen-Zhou, J.; Baldo, M. A.; Freedman, D. E.; Goldsmith, R. H.; Iii, T. G. Exploiting Chemistry an Molecular Systems for Quantum Information Science. Nat. Rev. Chem. 2020, 4, 490.

(27) Yu, C. J.; Krzyaniak, M. D.; Fataftah, M. S.; Wasielewski, M. R.; Freedman, D. E. A Concentrated Array of Copper Porphyrin Candidate Qubits. Chem. Sci. 2019, 10, 1702-1708.

(28) Urtizberea, A.; Natividad, E.; Alonso, P. J.; Andrés, M. A.; Gascón, I.; Goldmann, M.; Roubeau, O. A Porphyrin Spin Qubit and Its 2D Framework Nanosheets. Adv. Funct. Mater. 2018, 28, 1801695.

(29) Borges-Martínez, M.; Montenegro-Pohlhammer, N.; Yamamoto, Y.; Baruah, T.; Cárdenas-Jirón, G. Zn(II)-PorphyrinSquaraine Dyads as Potential Components for Dye-Sensitized Solar Cells: A Quantum Chemical Study of Optical and Charge Transport Properties. J. Phys. Chem. C 2020, 124, 12968-12981.

(30) Soman, R.; Sujatha, S.; Arunkumar, C. Semiconductive Properties of Zinc(II)-Porphyrinic Coordination Arrays. J. Porphyrins Phthalocyanines 2017, 21, 144-151.

(31) Xu, X.; Wang, S.; Yue, Y.; Huang, N. Semiconductive Porphyrin-Based Covalent Organic Frameworks for Sensitive NearInfrared Detection. ACS Appl. Mater. Interfaces 2020, 12, 3742737434.

(32) Perlovich, G. L.; Golubchikov, O. A.; Klueva, M. E. Thermodynamics of Porphyrin Sublimation. J. Porphyrins Phthalocyanines 2000, 04, 699-706.

(33) Cimatti, I.; Serrano, G.; Malavolti, L.; Cortigiani, B.; Betto, D.; Ouerghi, A.; Brookes, N. B.; Loth, S.; Mannini, M.; Totti, F.; et al. Vanadyl Phthalocyanines on Graphene/SiC(0001): Toward a Hybrid Architecture for Molecular Spin Qubits. Nanoscale Horiz 2019, 4, $1202-1210$.

(34) Malavolti, L.; Briganti, M.; Hänze, M.; Serrano, G.; Cimatti, I.; McMurtrie, G.; Otero, E.; Ohresser, P.; Totti, F.; Mannini, M.; et al. Tunable Spin-Superconductor Coupling of Spin 1/2 Vanadyl Phthalocyanine Molecules. Nano Lett. 2018, 18, 7955-7961.

(35) Grill, L.; Dyer, M.; Lafferentz, L.; Persson, M.; Peters, M. V.; Hecht, S. Nano-Architectures by Covalent Assembly of Molecular Building Blocks. Nat. Nanotechnol. 2007, 2, 687-691.

(36) Li, Y.; Xiao, J.; Shubina, T. E.; Chen, M.; Shi, Z.; Schmid, M.; Steinrück, H. P.; Gottfried, J. M.; Lin, N. Coordination and Metalation Bifunctionality of $\mathrm{Cu}$ with 5,10,15,20-Tetra(4- Pyridyl)Porphyrin: Toward a Mixed-Valence Two-Dimensional Coordination Network. J. Am. Chem. Soc. 2012, 134, 6401-6408.

(37) Escalera-Moreno, L.; Gaita-Ariño, A.; Coronado, E. Decoherence from Dipolar Interspin Interactions in Molecular Spin Qubits. Phys. Rev. B: Condens. Matter Mater. Phys. 2019, 100, 64405.

(38) Canarie, E. R.; Jahn, S. M.; Stoll, S. Quantitative StructureBased Prediction of Electron Spin Decoherence in Organic Radicals. J. Phys. Chem. Lett. 2020, 11, 3396-3400.

(39) Berliner, L. J.; Eaton, G. R.; Eaton, S. S. Distance Measurements in Biological Systems by EPR. In Biological Magnetic Resonance; Springer US: Boston, 2002; Vol. 19.

(40) Graham, M. J.; Yu, C. J.; Krzyaniak, M. D.; Wasielewski, M. R.; Freedman, D. E. Synthetic Approach To Determine the Effect of Nuclear Spin Distance on Electronic Spin Decoherence. J. Am. Chem. Soc. 2017, 139, 3196-3201.
(41) Albino, A.; Benci, S.; Tesi, L.; Atzori, M.; Torre, R.; Sanvito, S.; Sessoli, R.; Lunghi, A. First-Principles Investigation of Spin - Phonon Coupling in Vanadium-Based Molecular Spin Quantum Bits. Inorg. Chem. 2019, 58, 10260-10268.

(42) Lunghi, A.; Sanvito, S. How Do Phonons Relax Molecular Spins? Sci. Adv. 2019, 5, eaax7163.

(43) Jackson, C. E.; Lin, C. Y.; Johnson, S. H.; Van Tol, J.; Zadrozny, J. M. Nuclear-Spin-Pattern Control of Electron-Spin Dynamics in a Series of V(Iv) Complexes. Chem. Sci. 2019, 10, 8447-8454.

(44) Johnson, S. H.; Jackson, C. E.; Zadrozny, J. M. Programmable Nuclear-Spin Dynamics in Ti(IV) Coordination Complexes. Inorg. Chem. 2020, 59, 7479-7486.

(45) Mørkved, E. H.; Neset, S. M.; Kjøsen, H.; Hvistendahl, G.; Mo, F.; Balzarini, J.; Fransson, B.; Ragnarsson, U.; Francis, G. W. Template Assisted Cyclotetramerisations of 1,2,5-Thiadiazole-3,4Dicarbonitrile. Acta Chem. Scand. 1994, 48, 912-916.

(46) Stuzhin, P. A.; Bauer, E. M.; Ercolani, C. Tetrakis(Thiadiazole)Porphyrazines. 1. Syntheses and Properties of Tetrakis(Thiadiazole)Porphyrazine and Its Magnesium and Copper Derivatives. Inorg. Chem. 1998, 37, 1533-1539.

(47) Miyoshi, Y.; Takahashi, K.; Fujimoto, T.; Yoshikawa, H.; Matsushita, M. M.; Ouchi, Y.; Kepenekian, M.; Robert, V.; Donzello, M. P.; Ercolani, C.; et al. Crystal Structure, Spin Polarization, SolidState Electrochemistry, and High n-Type Carrier Mobility of a Paramagnetic Semiconductor: Vanadyl Tetrakis(Thiadiazole)Porphyrazine. Inorg. Chem. 2012, 51, 456-462.

(48) Hou, J.; Wang, Y.; Eguchi, K.; Nanjo, C.; Takaoka, T.; Sainoo, Y.; Awaga, K.; Komeda, T. Inter-Molecule Interaction for Magnetic Property of Vanadyl Tetrakis(Thiadiazole) Porphyrazine Film on $\mathrm{Au}\left(\begin{array}{lll}1 & 1 & 1\end{array}\right)$. Appl. Surf. Sci. 2018, 440, 16-19.

(49) Miyoshi, Y.; Kubo, M.; Fujinawa, T.; Suzuki, Y.; Yoshikawa, H.; Awaga, K. Electrochromism and Stable N-Type Doping of Highly Oriented Thin Films of Tetrakis(Thiadiazole)Porphyrazine. Angew. Chem., Int. Ed. 2007, 46, 5532-5536.

(50) Hou, J.; Wang, Y.; Eguchi, K.; Nanjo, C.; Takaoka, T.; Sainoo, Y.; Arafune, R.; Awaga, K.; Komeda, T. Enhanced Magnetic Spin-Spin Interactions Observed between Porphyrazine Derivatives on $\mathrm{Au}(111)$. Commun. Chem. 2020, 3, 1-11.

(51) Suzuki, Y.; Fujimori, M.; Yoshikawa, H.; Awaga, K. Packing Motifs and Magneto-Structural Correlations in Crystal Structures of Metallo-Tetrakis(1,2,5-Thiadiazole)Porphyrazine Series, MTTDPz $(\mathrm{M}=\mathrm{H}$ 2, $\mathrm{Fe}, \mathrm{Co}, \mathrm{Ni}, \mathrm{Cu}, \mathrm{Zn})$. Chem. - Eur. J. 2004, 10, 5158-5164.

(52) Hoshino, A.; Takenaka, Y.; Miyaji, H. Redetermination of the Crystal Structure of $\alpha$-Copper Phthalocyanine Grown on KCl. Acta Crystallogr., Sect. B: Struct. Sci. 2003, 59, 393-403.

(53) Linstead, R. P. Phthalocyanines. Part I. A New Type of Synthetic Colouring Matters. J. Chem. Soc. 1934, 1016.

(54) Bauer, E. M.; Cardarilli, D.; Ercolani, C.; Stuzhin, P. A.; Russo, U. Tetrakis(Thiadiazole)Porphyrazines. 2. Metal Complexes with $\mathrm{Mn}(\mathrm{II}), \mathrm{Fe}(\mathrm{II}), \mathrm{Co}(\mathrm{II}), \mathrm{Ni}(\mathrm{II})$, and $\mathrm{Zn}(\mathrm{II})$. Inorg. Chem. 1999, 38, 6114-6120.

(55) Greiner, S. P.; Rowlands, D. L.; Kreilick, R. W. EPR and ENDOR Study of Selected Porphyrin- and Phthalocyanine-Copper Complexes. J. Phys. Chem. 1992, 96, 9132-9139.

(56) Finazzo, C.; Calle, C.; Stoll, S.; Van Doorslaer, S.; Schweiger, A. Matrix Effects on Copper(II)Phthalocyanine Complexes. A Combined Continuous Wave and Pulse EPR and DFT Study. Phys. Chem. Chem. Phys. 2006, 8, 1942.

(57) Stoll, S.; Schweiger, A. EasySpin, a Comprehensive Software Package for Spectral Simulation and Analysis in EPR. J. Magn. Reson. 2006, 178, 42-55.

(58) Blumberg, W. E.; Peisach, J. An Electron Spin Resonance Study of Copper Uroporphyrin(III) and Other Touraco Feather Components. J. Biol. Chem. 1965, 240, 870-876.

(59) Gatteschi, D.; Sessoli, R.; Villain, J. Molecular Nanomagnets; Oxford University Press: Oxford, 2006.

(60) Casimir, H. B. G.; du Pré, F. K. Note on the Thermodynamic Interpretation of Paramagnetic Relaxation Phenomena. Physica 1938, 5, 507-511. 
(61) Cole, K. S.; Cole, R. H. Dispersion and Absorption in Dielectrics I. Alternating Current Characteristics. J. Chem. Phys. 1941, 9, 341-351.

(62) Eaton, S. S.; Eaton, G. R. Relaxation Mechanisms. eMagRes. 2016, 5, 1543-1556.

(63) Van Vleck, J. H. Paramagnetic Relaxation Times for Titanium and Chrome Alum. Phys. Rev. 1940, 57, 426-447.

(64) De Vroomen, A. C.; Lijphart, E. E.; Prins, D. Y. H.; Marks, J.; Poulis, N. J. Electron Spin-Lattice Relaxation of the Zeeman and Interaction Systems in $\mathrm{CuCs}_{2}\left(\mathrm{SO}_{4}\right)_{2} \cdot 6 \mathrm{H}_{2} \mathrm{O}$. Physica 1972, 61, 241249.

(65) Schweiger, A.; Jeschke, G. Principles of Pulse Electron Paramagnetic Resonance; Oxford University Press: Oxford, New York, 2001.

(66) Lin, C. Y.; Ngendahimana, T.; Eaton, G. R.; Eaton, S. S.; Zadrozny, J. M. Counterion Influence on Dynamic Spin Properties in a V(IV) Complex. Chem. Sci. 2019, 10, 548-555.

(67) Liddle, S. T.; Van Slageren, J. Improving F-Element Single Molecule Magnets. Chem. Soc. Rev. 2015, 44, 6655-6669.

(68) Atzori, M.; Benci, S.; Morra, E.; Tesi, L.; Chiesa, M.; Torre, R.; Sorace, L.; Sessoli, R. Structural Effects on the Spin Dynamics of Potential Molecular Qubits. Inorg. Chem. 2018, 57, 731-740.

(69) Lunghi, A.; Sanvito, S. The Limit of Spin Lifetime in Solid-State Electronic Spins. J. Phys. Chem. Lett. 2020, 11, 6273-6278.

(70) Lunghi, A. Ligand-Field Contributions to Spin-Phonon Coupling in a Family of Vanadium Molecular Qubits from MultiReference Electronic Structure Theory. 2019, arXiv: 1912.4545 [cond-mat.mtrl-sci].

(71) Escalera-Moreno, L.; Suaud, N.; Gaita-Ariño, A.; Coronado, E. Determining Key Local Vibrations in the Relaxation of Molecular Spin Qubits and Single-Molecule Magnets. J. Phys. Chem. Lett. 2017, 8, 1695-1700.

(72) Lunghi, A.; Totti, F.; Sanvito, S.; Sessoli, R. Intra-Molecular Origin of the Spin-Phonon Coupling in Slow-Relaxing Molecular Magnets. Chem. Sci. 2017, 8, 6051-6059.

(73) Breuer, H. P.; Petruccione, F. The Theory of Open Quantum Systems; Oxford University Press: New York, 2007.

(74) Redfield, A.G. The Theory of Relaxation Processes. Adv. Magn. Opt. Reson. 1965, 1, 1.

(75) Penchoff, D. Group Theory: An Application of Discrete Groups; http://sces.phys.utk.edu/ moreo/mm08/penchoff. pdfhttp://sces.phys.utk.edu/ moreo/mm08/penchoff.pdf.

(76) Mirzoyan, R.; Hadt, R. G. The Dynamic Ligand Field of a Molecular Qubit: Decoherence through Spin-Phonon Coupling. Phys. Chem. Chem. Phys. 2020, 22, 11249-11265.

(77) Bader, K.; Winkler, M.; van Slageren, J. Tuning of Molecular Qubits: Very Long Coherence and Spin-Lattice Relaxation Times. Chem. Commun. 2016, 52, 3623-3626.

(78) Macrae, C. F.; Sovago, I.; Cottrell, S. J.; Galek, P. T. A.; McCabe, P.; Pidcock, E.; Platings, M.; Shields, G. P.; Stevens, J. S.; Towler, M.; et al. Mercury 4.0: From Visualization to Analysis, Design and Prediction. J. Appl. Crystallogr. 2020, 53, 226-235.

(79) Neese, F. The ORCA Program System. Wiley Interdiscip. Rev.: Comput. Mol. Sci. 2012, 2, 73.

(80) Hutter, J.; Iannuzzi, M.; Schiffmann, F.; VandeVondele, J. Cp2k: Atomistic Simulations of Condensed Matter Systems. Wiley Interdiscip. Rev. Comput. Mol. Sci. 2014, 4, 15-25.

(81) Califano, S.; Schettino, V.; Neto, N. Lattice Dynamics of Molecular Crystals; Springer-Verlag: Berlin Heidelberg, 1981.

(82) Vandevondele, J.; Krack, M.; Mohamed, F.; Parrinello, M.; Chassaing, T.; Hutter, J. Quickstep: Fast and Accurate Density Functional Calculations Using a Mixed Gaussian and Plane Waves Approach. Comput. Phys. Commun. 2005, 167, 103-128.

(83) Perdew, J. P. Density-Functional Approximation for the Correlation Energy of the Inhomogeneous Electron Gas. Phys. Rev. B: Condens. Matter Mater. Phys. 1986, 33, 8822-8824.

(84) Perdew, J. P.; Burke, K.; Ernzerhof, M. Generalized Gradient Approximation Made Simple. Phys. Rev. Lett. 1996, 77, 3865.
(85) Bradley, C. J.; Cracknell, A. P. The Mathematical Theory of Symmetry in Solids Representation Theory for Point Groups and Space Groups; Clarendon Press: Oxford, 1972.

(86) Iannone, F.; Ambrosino, F.; Bracco, G.; De Rosa, M.; De Funel, A.; Guarnieri, G.; Migliori, S.; Palombi, F.; Ponti, G.; Santomauro, G.; Procacci, P. CRESCO ENEA HPC clusters: a working example of a multifabric GPFS Spectrum Scale layout. 2019 International Conference on High Performance Computing \& Simulation (HPCS), Dublin; IEEE, 2019; p p 1051-1052, DOI: 10.1109/ HPCS48598.2019.9188135. 